\title{
Hadronic contributions to the muon anomaly in the constituent chiral quark model
}

\author{
David Greynat $^{a}$ and Eduardo de Rafael ${ }^{b, 1}$ \\ ${ }^{a}$ Departamento de Fúsica Teórica, Facultad de Ciencias, Universidad de Zaragoza, \\ E-50009 Zaragoza, Spain \\ ${ }^{b}$ Centre de Physique Théorique, ${ }^{2}$ \\ CNRS-Luminy, Case 907, F-13288 Marseille Cedex 9, France \\ E-mail: david.greynat@gmail.com, EdeR@cpt.univ-mrs.fr
}

ABSTRACT: The hadronic contributions to the anomalous magnetic moment of the muon which are relevant for the confrontation between theory and experiment at the present level of accuracy, are evaluated within the same framework: the constituent chiral quark model. This includes the contributions from the dominant hadronic vacuum polarization as well as from the next-to-leading order hadronic vacuum polarization, the contributions from the hadronic light-by-light scattering, and the contributions from the electroweak hadronic $Z \gamma \gamma$ vertex. They are all evaluated as a function of only one free parameter: the constituent quark mass. We also comment on the comparison between our results and other phenomenological evaluations.

KeYwords: Phenomenological Models, NLO Computations

\footnotetext{
${ }^{1}$ Partially based on the talk of EdeR at the INT Workshop on The hadronic light-by-light contribution to the muon anomaly, February 28-March 4 (2011).

${ }^{2}$ Unité Mixte de Recherche (UMR7332) du CNRS et des Universités Aix Marseille 1, Aix Marseille 2 et Sud Toulon-Var, affiliée à la FRUMAM.
} 


\section{Contents}

1 Introduction 1

2 Hadronic vacuum polarization 3

$\begin{array}{lll}3 & \text { Hadronic vacuum polarization contributions at next-to-leading order } & \mathbf{7}\end{array}$

3.1 Class A: HVP insertions in the fourth order QED vertex diagrams $\quad 7$

3.2 Class B: HVP insertions in the QED vertex with an electron loop 8

$\begin{array}{ll}3.3 \text { Class C: iterated HVP contributions } & 10\end{array}$

3.4 Class D: contributions with HVP corrections at $\mathcal{O}(\alpha) \quad 13$

$\begin{array}{lll}\text { 3.4.1 Contribution from the } \pi^{0} \gamma \text { intermediate state } & 14\end{array}$

$\begin{array}{ll}\text { 3.4.2 Contribution from the quark loop to } \mathcal{O}(\alpha) & 18\end{array}$

4 Hadronic light-by-light scattering contributions $\quad 19$

4.1 Class A: the constituent quark loop contribution 21

$\begin{array}{ll}\text { 4.2 Class B: the } \pi^{0} \text { exchange contribution } & 22\end{array}$

5 Hadronic electroweak contributions $\quad 25$

$\begin{array}{llr}6 & \text { Summary and conclusions } & 28\end{array}$

\section{Introduction}

The present experimental world average of the anomalous magnetic moment of the muon $a_{\mu}$, assuming CPT-invariance, viz. $a_{\mu^{+}}=a_{\mu^{-}}$, is

$$
a_{\mu}^{(\exp )}=116592080(63) \times 10^{-11} \quad(0.54 \mathrm{ppm}),
$$

where the total uncertainty includes a $0.46 \mathrm{ppm}$ statistical uncertainty and a $0.28 \mathrm{ppm}$ systematic uncertainty, combined in quadrature. This result is largely dominated by the latest series of precise measurements carried out at the Brookhaven National Laboratory (BNL) by the E821 collaboration, with results reported in ref. [1] and references therein. The prediction of the Standard Model, as a result of contributions from many physicists is ${ }^{1}$

$$
a_{\mu}^{(\mathrm{SM})}=116591801(49) \times 10^{-11},
$$

where the error here is dominated at present by the lowest order hadronic vacuum polarization contribution uncertainty [4] $\left( \pm 42.0 \times 10^{-11}\right)$, as well as by the contribution from the hadronic light-by-light scattering, which is theoretically estimated to be $(105 \pm 26) \times$

\footnotetext{
${ }^{1}$ For recent reviews see e.g. refs. [2, 3] and references therein.
} 
$10^{-11}[5]$. The results quoted in (1.1) and (1.2) imply a significant 3.6 standard deviation between theory and experiment which deserves attention. In order to firmly attribute this discrepancy to new Physics, one would like to reduce the theoretical uncertainties as much as possible, parallel to the new experimental efforts towards an even more precise measurement of $a_{\mu}$ in the near future [6,7]. It is therefore important to reexamine critically the various theoretical contributions to eq. (1.2); in particular the hadronic contributions. Ideally, one would like to do that within the framework of Quantum Chromodynamics(QCD). Unfortunatley, this demands mastering QCD at all scales from short to long distances, something which is not under full analytic control at present. Therefore, one has to resort to experimental information whenever possible, to QCD inspired hadronic models, and to lattice QCD simulations which are as yet at an early stage. As a result, all the theoretical evaluations of the hadronic contributions to $a_{\mu}$ have systematic errors which are not easy to pin down rigorously.

Our purpose here is to establish a simple reference model to evaluate the various hadronic contributions to $a_{\mu}$ within the same framework, and use it as a yardstick to compare with the more detailed evaluations in the literature. The reference model which we propose is based on the Constituent Chiral Quark Model $(\mathrm{C} \chi \mathrm{QM})$ [8]. This model emerged as an attempt to reconcile the successes of phenomenological quark models, like the De Rújula-Georgi-Glashow model [9], with QCD. The corresponding Lagrangian proposed by Manohar and Georgi (MG) is an effective field theory which incorporates the interactions of the low-lying pseudoscalar particles of the hadronic spectrum, the NambuGoldstone modes of the spontaneously broken chiral symmetry ( $\mathrm{S} \chi \mathrm{SB}$ ), to lowest order in the chiral expansion [10] and in the presence of chirally rotated quark fields. Because

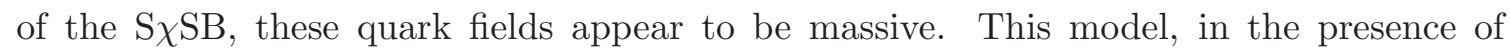
$\mathrm{SU}(3)_{L} \times \mathrm{SU}(3)_{R}$ external sources has been reconsidered recently by one of us [11]. As emphasized by Weinberg [12], the corresponding effective Lagrangian is renormalizable in the Large- $\mathrm{N}_{\mathrm{c}}$ limit; however, the number of the required counterterms depends crucially on the value of the coupling constant $g_{A}$ in the model and, as shown in [11], it is minimized for $g_{A}=1$. With this choice, and a value for the constituent quark mass fixed phenomenologically, the model reproduces rather well the values of several well known low energy constants.

As discussed in ref. [11] the $\mathrm{C} \chi \mathrm{QM}$ model has, however, its own limitations. Applications to the evaluation of low-energy observables involving the integration of Green's functions over a full range of euclidean momenta fail, in general, because there is no matching of the model to the QCD short-distance behaviour. There is, however, an exceptional class of low-energy observables for which the MG-Lagrangian predictions can be rather reliable. This is the case when the leading short-distance behaviour of the underlying Green's function of a given observable is governed by perturbative QCD. The decay $\pi^{0} \rightarrow e^{+} e^{-}$, which was discussed in ref. [11], is one such example. Other interesting examples of this class of observables are the contributions to $a_{\mu}$ from Hadronic Vacuum Polarization, from the Hadronic Light-by-Light Scattering and from the Hadronic $Z \gamma \gamma$ vertex ( provided, as we shall see, that the coupling $g_{A}$ is fixed to $g_{A}=1$ ). The evaluation of these contributions with the $\mathrm{C} \chi \mathrm{QM}$ Lagrangian is the main purpose of this paper and they are discussed below 


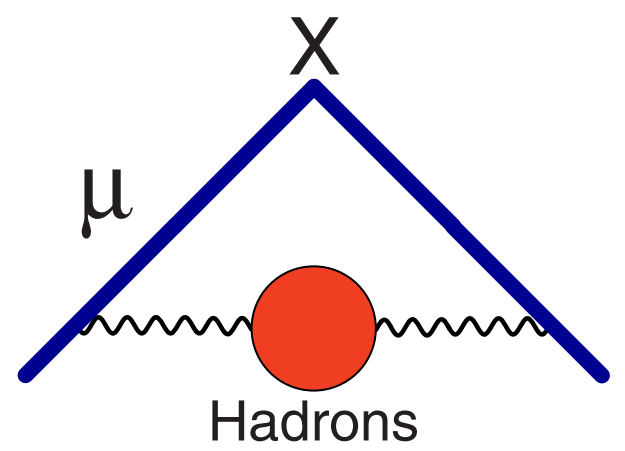

Figure 1. Hadronic Vacuum Polarization contribution to the muon anomaly.

in detail. They have the advantage of simplicity and can provide a consistency check with the more elaborated phenomenological approaches.

\section{Hadronic vacuum polarization}

There is a well known representation [13] of the dominant contribution to the muon anomaly from the hadronic vacuum polarization shown in figure 1

$$
\frac{1}{2}\left(g_{\mu}-2\right)_{\mathrm{HVP}} \equiv a_{\mu}^{(\mathrm{HVP})}=\int_{4 M_{Q}^{2}}^{\infty} \frac{d t}{t} K\left(\frac{t}{m_{\mu}^{2}}\right) \frac{1}{\pi} \operatorname{Im} \Pi^{(\mathrm{HVP})}(t),
$$

where $^{2}$

$$
K\left(\frac{t}{m_{\mu}^{2}}\right)=\left(\frac{\alpha}{\pi}\right) \int_{0}^{1} d x \frac{x^{2}(1-x)}{x^{2}+\frac{t}{m_{\mu}^{2}}(1-x)},
$$

and $\frac{1}{\pi} \operatorname{Im} \Pi(t)$ denotes the electromagnetic hadronic spectral function. It is a useful representation because of the direct relation to the one-photon $e^{+} e^{-}$annihilation cross-section into hadrons $\left(m_{e} \rightarrow 0\right)$ :

$$
\sigma(t)_{\left\{e^{+} e^{-} \rightarrow(\gamma) \rightarrow \text { hadrons }\right\}}=\frac{4 \pi^{2} \alpha}{t} \frac{1}{\pi} \operatorname{Im} \Pi^{(\mathrm{HVP})}(t),
$$

and hence to experimental data, provided the necessary radiative corrections have been made to insure that one is using the one-photon cross-section.

In the $\mathrm{C} \chi \mathrm{QM}$ with active $u, d, s$ quarks and, to a first approximation, with neglect of gluonic corrections

$$
\begin{aligned}
\frac{1}{\pi} \operatorname{Im} \Pi^{(\mathrm{HVP})}(t)= & \left(\frac{\alpha}{\pi}\right) N_{c}\left[\left(\frac{2}{3}\right)^{2}+\left(-\frac{1}{3}\right)^{2}+\left(-\frac{1}{3}\right)^{2}\right] \\
& \times \delta\left(\frac{1}{2}-\frac{1}{6} \delta^{2}\right) \theta\left(t-4 M_{Q}^{2}\right), \quad \text { with } \delta=\sqrt{1-\frac{4 M_{Q}^{2}}{t}} .
\end{aligned}
$$

\footnotetext{
${ }^{2}$ The analytic expression of $K\left(t / m_{\mu}^{2}\right)$ was first given in ref. [14]; see also ref. [19].
} 
The integral in eq. (2.1) can then be easily done with the result shown in figure 3, the curve labeled (a), where the value for $a_{\mu}^{(\mathrm{HVP})}$ is plotted as a function of the only free parameter in the model, the constituent quark mass $M_{Q} \cdot{ }^{3}$

The constituent quark fields in the $\mathrm{C} \chi \mathrm{QM}$ are assumed to have gluonic interactions as well but, since the Goldstone modes are already in the Lagrangian, the color-SU(3) coupling constant is supposed to be no longer running below a scale $\mu_{0} \simeq 2 \mathrm{GeV}$ where $\alpha_{s}\left(\mu_{0}\right) \simeq 0.33$ and non-perturbative effects become significant. With inclusion of the leading gluonic corrections in perturbation theory, and to leading order in Large- $\mathrm{N}_{\mathrm{c}}$, the spectral function in eq. (2.4) then becomes

$$
\begin{aligned}
\frac{1}{\pi} \operatorname{Im} \Pi^{(\mathrm{HVP})}(t)=\left(\frac{\alpha}{\pi}\right) & N_{c} \frac{2}{3}\left\{\delta\left(\frac{1}{2}-\frac{1}{6} \delta^{2}\right)\right. \\
& \left.+\left[\frac{N_{c} \alpha_{\mathrm{S}}\left(\mu_{0}\right)}{\pi} \frac{3}{8} \theta\left(\mu_{0}^{2}-t\right)+\frac{N_{c} \alpha_{\mathrm{s}}(\sqrt{t})}{\pi} \frac{3}{8} \theta\left(t-\mu_{0}^{2}\right)\right] \rho_{\mathrm{KS}}(t)\right\},
\end{aligned}
$$

where $\rho_{\mathrm{KS}}(t)$ can be extracted from the early QED calculation of Kllen and Sabry [18] (see also ref. [19]):

$$
\begin{aligned}
\rho_{\mathrm{KS}}(t)= & \delta\left(\frac{5}{8}-\frac{3}{8} \delta^{2}-\left(\frac{1}{2}-\frac{1}{6} \delta^{2}\right) \log \left[64 \frac{\delta^{4}}{\left(1-\delta^{2}\right)^{3}}\right]\right) \\
& +\left(\frac{11}{16}+\frac{11}{24} \delta^{2}-\frac{7}{48} \delta^{4}+\left(\frac{1}{2}+\frac{1}{3} \delta^{2}-\frac{1}{6} \delta^{4}\right) \log \left[\frac{(1+\delta)^{3}}{8 \delta^{2}}\right]\right) \log \left[\frac{1+\delta}{1-\delta}\right] \\
& +2\left(\frac{1}{2}+\frac{1}{3} \delta^{2}-\frac{1}{6} \delta^{4}\right)\left(2 \operatorname{Li}_{2}\left[\frac{1-\delta}{1+\delta}\right]+\operatorname{Li}_{2}\left[-\frac{1-\delta}{1+\delta}\right]\right) .
\end{aligned}
$$

Also, at the level of the accuracy expected from the $\mathrm{C} \chi \mathrm{QM}$, it is sufficient to use the one loop expression

$$
\frac{\alpha_{\mathrm{S}}(\sqrt{t})}{\pi} \simeq \frac{1}{\left(\frac{11}{6} N_{c}-\frac{n_{f}}{3}\right) \log \frac{\sqrt{t}}{\Lambda}}, \quad \text { with } \quad \Lambda \simeq 250 \mathrm{MeV} \quad \text { and } \quad n_{f}=3
$$

The resulting value for $a_{\mu}^{(\mathrm{HVP})}$ in eq. (2.1) in $10^{-10}$ units versus $M_{Q}$ in $\mathrm{MeV}$ is shown in figure 3, the curved labeled (b).

In order to compare the $\mathrm{C} \chi \mathrm{QM}$ results for $a_{\mu}^{(\mathrm{HVP})}$ with the phenomenological determinations which incorporate experimental data, we still have to correct for the fact that the curve (b) in figure 3 only reflects the Large- $\mathrm{N}_{\mathrm{c}}$ estimate of the model. As an estimate of

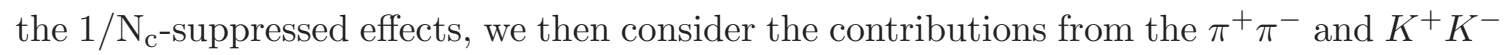
intermediate states to the spectral function in eq. (2.1), as predicted by the $\mathrm{C} \chi \mathrm{QM}$. Notice that in this evaluation, the point like coupling $(-i e)\left(p^{\mu}-p^{\prime \mu}\right)$ of scalar QED is replaced by the dressed coupling:

$$
(-i e)\left(p^{\mu}-p^{\prime \mu}\right) \Rightarrow(-i e)\left(p^{\mu}-p^{\prime \mu}\right)\left\{1+\mathcal{F}\left(Q^{2}\right)\right\},
$$

${ }^{3}$ There are many estimates of this contribution, as well as some of the higher order ones, with quark models which can be found in the literature. An earlier reference is [15] and two more recent ones [16] and [17]. 

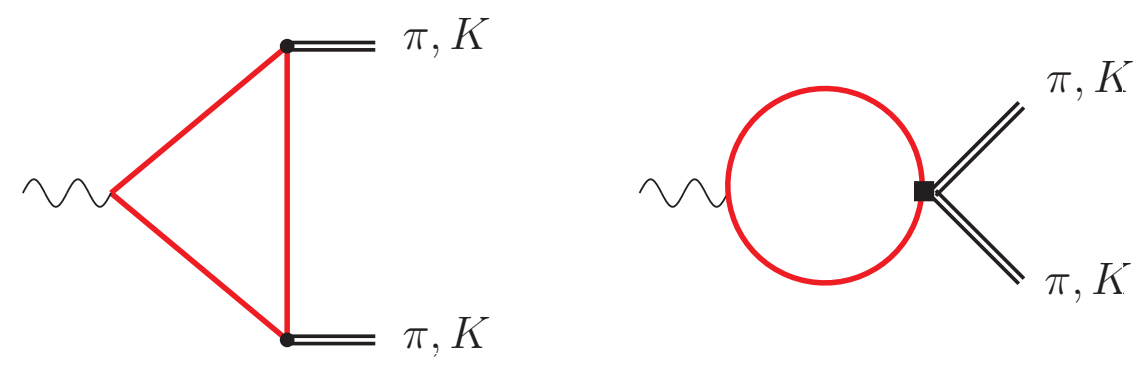

Figure 2. Feynman diagrams contributing to the electromagnetic form factor $\mathcal{F}\left(Q^{2}\right)$ in eq. (2.9).

with $\mathcal{F}\left(Q^{2}\right)$ the pion (kaon) electromagnetic form factor of the $\mathrm{C} \chi \mathrm{QM}$, at the one loop level in figure 2 which, for $g_{A}=1$, is given by the expression:

$$
\begin{aligned}
\mathcal{F}\left(Q^{2}\right) & =\mathrm{N}_{\mathrm{c}} \frac{Q^{2}}{16 \pi^{2} f_{\pi}^{2}} \int_{0}^{1} d x x \int_{0}^{1} d y[1-x(1-y)] \frac{M_{Q}^{2}}{M_{Q}^{2}+x y(1-x) Q^{2}-i \epsilon} \\
& =\frac{\mathrm{N}_{\mathrm{c}}}{16 \pi^{2}} \frac{Q^{2}}{f_{\pi}^{2}}\left(-\frac{4 M_{Q}^{2}}{Q^{2}}\right)\left[1+\frac{1}{2} \sqrt{1+\frac{4 M_{Q}^{2}}{Q^{2}}} \log \frac{\sqrt{1+\frac{4 M_{Q}^{2}}{Q^{2}}}-1}{\sqrt{1+\frac{4 M_{Q}^{2}}{Q^{2}}}+1}\right] .
\end{aligned}
$$

In fact, this form factor, for $g_{A} \neq 1$, has UV-contributions which diverge and would require an explicit counterterm in the Lagrangian. The form factor in eq. (2.9) has the asymptotic behaviour:

$$
\lim _{Q^{2} \rightarrow 0} \mathcal{F}\left(Q^{2}\right)=\frac{\mathrm{N}_{\mathrm{c}}}{16 \pi^{2} f_{\pi}^{2}} Q^{2}\left[\frac{1}{3}+\frac{1}{30} \frac{Q^{2}}{M_{Q}^{2}}+\mathcal{O}\left(\frac{Q^{4}}{M_{Q}^{4}}\right)\right],
$$

and, in particular, fixes the value of the coupling constant $L_{9}$ in the $\chi \mathrm{PT}$ effective Lagrangian to the value [20]:

$$
L_{9}=\frac{\mathrm{N}_{\mathrm{c}}}{16 \pi^{2}} \frac{1}{3} .
$$

Also, for $Q^{2}=-t$ and $t \geq 4 M_{Q}^{2}$ the form factor develops an imaginary part:

$$
\frac{1}{\pi} \operatorname{Im} \mathcal{F}(t)=\frac{\mathrm{N}_{\mathrm{c}}}{16 \pi^{2}}\left(-\frac{2 M_{Q}^{2}}{f_{\pi}^{2}}\right) \sqrt{1-\frac{4 M_{Q}^{2}}{t}} \theta\left(t-4 M_{Q}^{2}\right),
$$

and the form factor $\mathcal{F}\left(Q^{2}\right)$ obeys a once subtracted dispersion relation

$$
\mathcal{F}\left(Q^{2}\right)=\int_{4 M_{Q}^{2}}^{\infty}\left(\frac{1}{t+Q^{2}-i \epsilon}-\frac{1}{t}\right) \frac{1}{\pi} \operatorname{Im} \mathcal{F}(t)
$$

the subtraction ensuring that $\mathcal{F}\left(Q^{2}=0\right)=0$, as fixed by lowest order $\chi \mathrm{PT}$.

The form factor $\mathcal{F}\left(Q^{2}\right)$, however, does not match the QCD behaviour at large- $Q^{2}$ values and, therefore, the estimate we propose for the $1 / \mathrm{N}_{\mathrm{c}}$-suppresed contributions to the the muon anomaly can only be considered as reasonable up to values of $t$ in eq. (2.1) below 


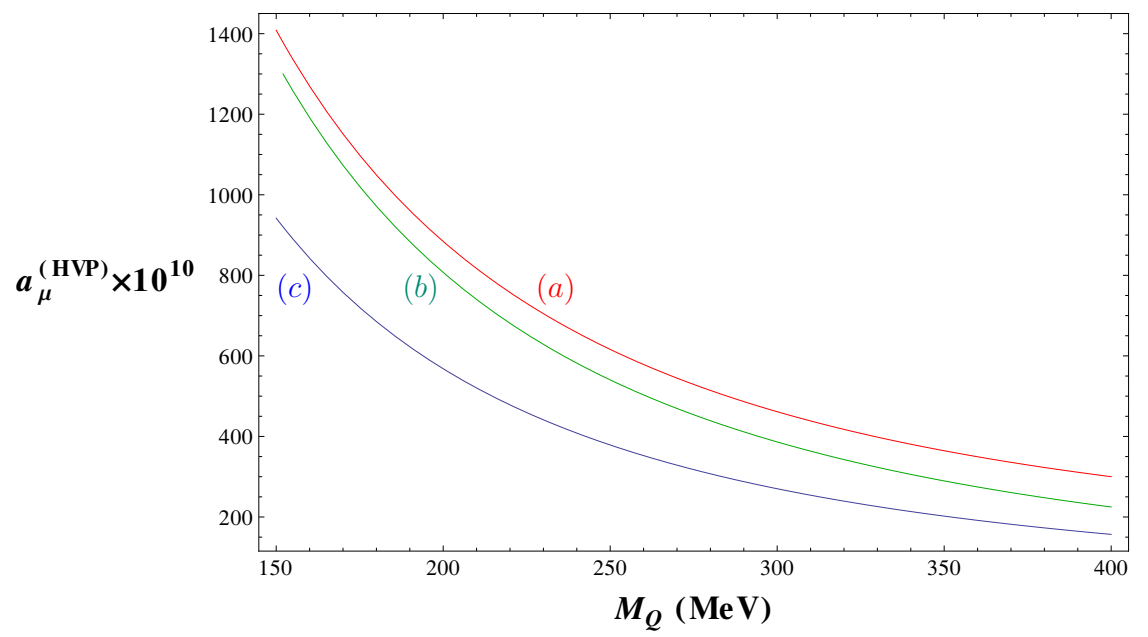

Figure 3. $a_{\mu}^{(\mathrm{HVP})}$ in the C $\chi \mathrm{QM}$. Curve (a) is the contribution using the spectral function in eq. (2.4); curve (b) the contribution using the corrected spectral function in eq. (2.5) and curve (c) the contribution using the corrected spectral function in eq. (2.5) with subleading $\pi^{+} \pi^{-}$and $K^{+} K^{-}$contributions incorporated as discussed in the text.

$t \sim \mu_{0}$ where the asymptotic pQCD regime sets in. Contributions beyond $t \sim \mu_{0}$ have already been taken into account by the second term of the spectral function in eq. (2.5).

The total contribution to $a_{\mu}^{(\mathrm{HVP})}$ in the $\mathrm{C} \chi \mathrm{QM}$, which incorporates gluonic contributions in the spectral function in eq. (2.5) as well as the subleading $\pi^{+} \pi^{-}$and $K^{+} K^{-}$ contributions in the way described above is shown in figure 3 as a function of $M_{Q}$, the curve labeled (c).

These considerations provide us with a framework to fix the constituent quark mass $M_{Q}$. The prediction of the $\mathrm{C} \chi \mathrm{QM}$, as described above, should be compared to the phenomenological contribution from hadrons formed of $u, d$ and $s$ quarks only, at the level of one-photon exchange. Contributions like for example the one from an intermediate $\pi^{0} \gamma$ state should therefore be excluded so far (more on that later on), as well as those involving $c, b$ and $t$ quarks. From the numbers quoted in table 2 of ref. [4], we then find that this restriction reduces the phenomenological determination of the anomaly from hadronic vacuum polarization to a central value

$$
\left.a_{\mu}^{(\mathrm{HVP})}\right|_{\text {phen. }} \simeq 653 \times 10^{-10}
$$

which, when compared with the results plotted in figure 3 , shows that fixing $M_{Q}$ in the range

$$
M_{Q}=(240 \pm 10) \mathrm{MeV},
$$

reproduces the phenomenological determination within an error of less than $10 \%$. This determination of the constituent quark mass is the value which we shall systematically use for $M_{Q}$ when evaluating the predictions for the other hadronic contributions to the muon anomaly. We shall then compare them to the various phenomenological determinations in the literature. 
We wish to emphasize, however, that the error of $10 \mathrm{MeV}$ in eq. (2.15) only reflects the phenomenological choice that we have made in order to fix $M_{Q}$. As discussed in the Introduction the $\mathrm{C} \chi \mathrm{QM}$ is only a model of low energy QCD and, as such, there is no a priori way to fix $M_{Q}$ from first principles. The error in eq. (2.15) does not reflect the systematic error due to other plausible ways of fixing $M_{Q}$.

At this stage we wish to point out that the recent lattice QCD determination of $a_{\mu}^{(\mathrm{HVP})}$ with two flavours reported in ref. [21] can also be very well digested with a value of $M_{Q}$ within the range given in eq. (2.15).

\section{Hadronic vacuum polarization contributions at next-to-leading order}

The Hadronic Vacuum Polarization contributions at $\mathcal{O}\left(\frac{\alpha}{\pi}\right)^{3}$ were classified long time ago in ref. [15]. Let us discuss their evaluation in the $\mathrm{C} \chi \mathrm{QM}$.

\subsection{Class A: HVP insertions in the fourth order QED vertex diagrams}

They correspond to the Feynman diagrams shown in figure 4, where the diagrams in each line in this figure are well-defined gauge invariant subsets. Here, the equivalent of the function $K\left(t / m_{\mu}^{2}\right)$ in eq. (2.2) at the two loop level, which we call $K^{(4)}\left(t / m_{\mu}^{2}\right)$, was calculated analytically by Barbieri and Remiddi [22]. The exact expression is, however, rather cumbersome and for our purposes it is more convenient to use an expansion of this function in powers of $\frac{m_{\mu}^{2}}{t}$, which is justified by the fact that the hadronic threshold in the integral that gives the contribution from the diagrams of Class A:

$$
a_{\mu}^{(\mathrm{HVP}-\mathrm{A})}=\int_{4 M_{Q}^{2}}^{\infty} \frac{d t}{t} K^{(4)}\left(t / m_{\mu}^{2}\right) \frac{1}{\pi} \operatorname{Im} \Pi^{(\mathrm{HVP})}(t),
$$

starts at $4 M_{Q}^{2} \gg m_{\mu}^{2}$. The terms in the expansion in question for the kernel $K^{(4)}\left(t / m_{\mu}^{2}\right)$ which we have retained are:

$$
\begin{aligned}
K^{(4)}\left(t / m_{\mu}^{2}\right)=\left(\frac{\alpha}{\pi}\right)^{2} & \left(-2 \frac{m_{\mu}^{2}}{t}\right)\left\{\left(\frac{23}{36} \log \frac{t}{m_{\mu}^{2}}-\frac{223}{54}+\frac{\pi^{2}}{3}\right)\right. \\
& +\frac{m_{\mu}^{2}}{t}\left(-\frac{19}{144} \log ^{2} \frac{t}{m_{\mu}^{2}}+\frac{367}{216} \log \frac{t}{m_{\mu}^{2}}-\frac{8785}{1152}+\frac{37}{48} \pi^{2}\right) \\
& +\left(\frac{m_{\mu}^{2}}{t}\right)^{2}\left(-\frac{141}{80} \log ^{2} \frac{t}{m_{\mu}^{2}}+\frac{10079}{3600} \log \frac{t}{m_{\mu}^{2}}-\frac{13072841}{432000}+\frac{883}{240} \pi^{2}\right) \\
& +\left(\frac{m_{\mu}^{2}}{t}\right)^{3}\left(-\frac{961}{80} \log ^{2} \frac{t}{m_{\mu}^{2}}+\frac{6517}{1800} \log \frac{t}{m_{\mu}^{2}}-\frac{2034703}{16000}+\frac{1301}{80} \pi^{2}\right) \\
& \left.+\mathcal{O}\left[\left(\frac{m_{\mu}^{2}}{t}\right)^{4}\right]\right\}
\end{aligned}
$$

Using the $\mathrm{C} \chi \mathrm{QM}$ spectral function in eq. (2.5), we find for this contribution the following result:

$$
a_{\mu}^{(\mathrm{HVP}-\mathrm{A})}\left(M_{Q}=240 \mathrm{MeV}\right)=-171 \times 10^{-11},
$$



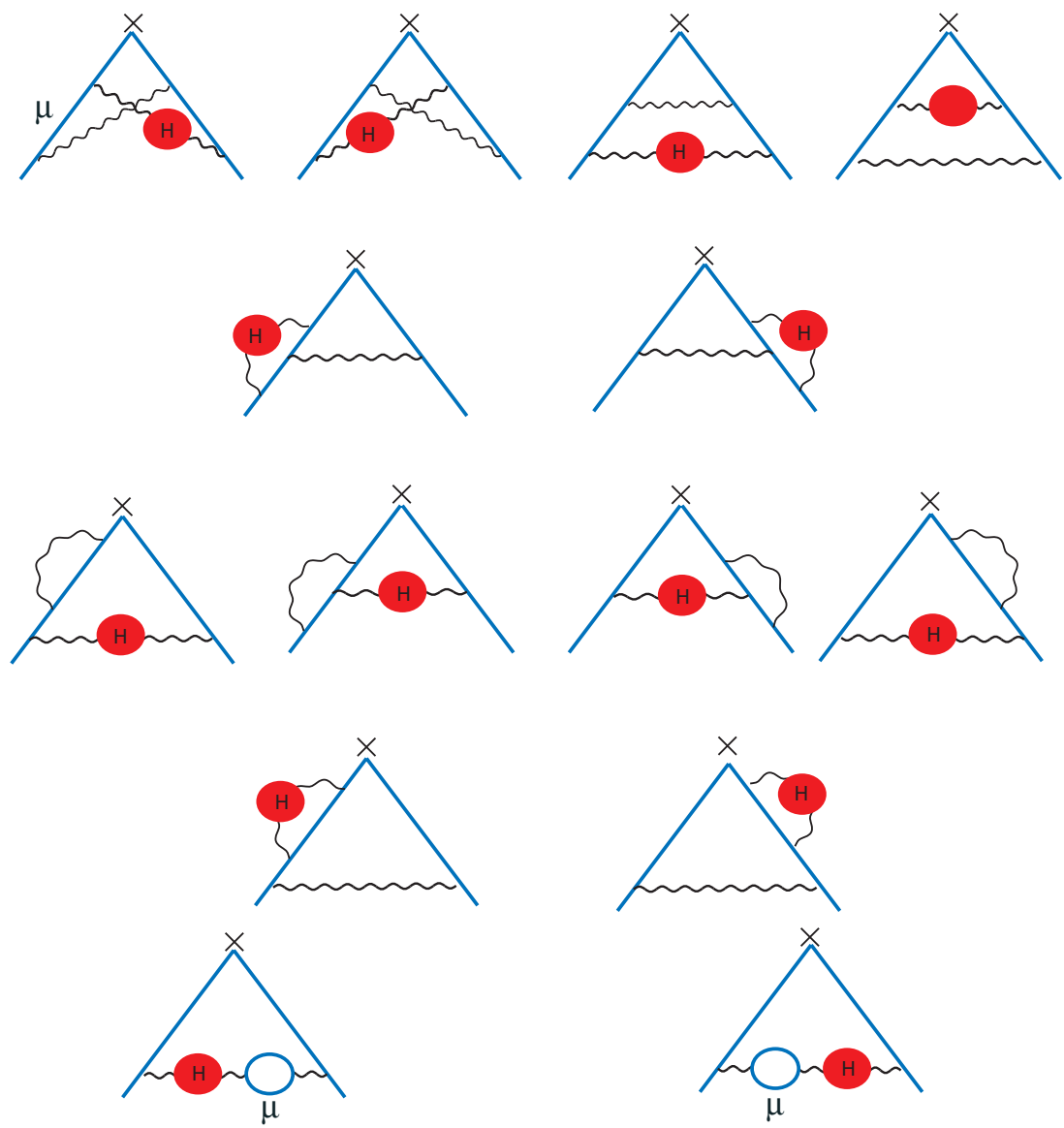

Figure 4. Feynman diagrams corresponding to the Class A contribution to the muon anomaly.

with a range

$$
-181 \times 10^{-11} \leq a_{\mu}^{(\mathrm{HVP}-\mathrm{A})} \leq-161 \times 10^{-11}, \quad \text { for } \quad 230 \mathrm{MeV} \leq M_{Q} \leq 250 \mathrm{MeV} .
$$

This result is to be compared with the phenomenological determination [23]:

$$
a_{\mu}^{(\mathrm{HVP}-\mathrm{A})}=-(207.3 \pm 1.9) \times 10^{-11} .
$$

We conclude that the $\mathrm{C} \chi \mathrm{QM}$ reproduces, within the expected accuracy of the model, this phenomenological value, specially if we take into account that the phenomenological determination includes contributions subleading in $1 / \mathrm{N}_{\mathrm{c}}$ and from higher flavours, which are beyond the duality domain of the model.

\subsection{Class B: HVP insertions in the QED vertex with an electron loop}

This is the contribution from the two Feynman diagrams in figure 5. A convenient representation [15] for this contribution, is the one given by the integral

$$
\begin{aligned}
a_{\mu}^{(\mathrm{HVP}-\mathrm{B})}= & \left(\frac{\alpha}{\pi}\right)^{2} \int_{4 M_{Q}^{2}}^{\infty} \frac{d t}{t} \int_{0}^{1} d x \frac{x^{2}(1-x)}{x^{2}+\frac{t}{m_{\mu}^{2}}(1-x)} \\
& \times\left[-2 \operatorname{Re} \Pi^{(e)}\left(-\frac{x^{2}}{1-x} m_{\mu}^{2}\right) \frac{1}{\pi} \operatorname{Im} \Pi^{(\mathrm{HVP})}(t)\right],
\end{aligned}
$$




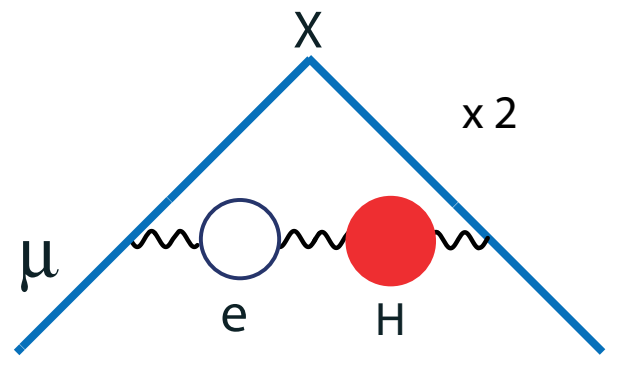

Figure 5. Feynman diagrams corresponding to the Class B contribution to the muon anomaly.

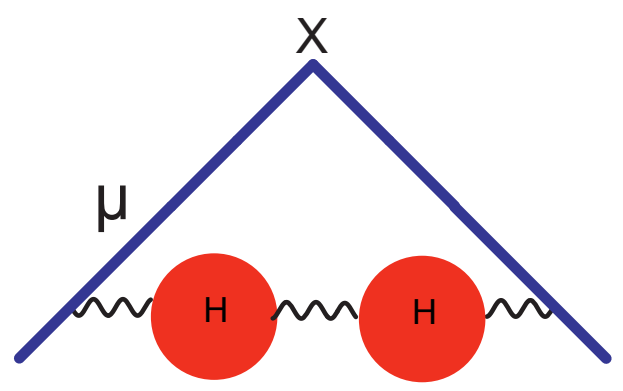

Figure 6. HVP contributions at $\mathcal{O}(\alpha)$.

where

$$
\operatorname{Re} \Pi^{(e)}\left(-\frac{x^{2}}{1-x} m_{\mu}^{2}\right)=\frac{8}{9}-\frac{1}{3} \beta^{2}+\beta\left(\frac{1}{2}-\frac{1}{6} \beta^{2}\right) \log \left(\frac{-1+\beta}{1+\beta}\right)
$$

with

$$
\beta=\sqrt{1+4 \frac{1-x}{x^{2}} \frac{m_{e}^{2}}{m_{\mu}^{2}}},
$$

denotes the real part of the electron self-energy. Using the $\mathrm{C} \chi \mathrm{QM}$ spectral function in eq. (2.5), we find for this contribution the following results:

$$
a_{\mu}^{(\mathrm{HVP}-\mathrm{B})}\left(M_{Q}=240 \mathrm{MeV}\right)=88.9 \times 10^{-11},
$$

with a range

$$
82.6 \times 10^{-11} \leq a_{\mu}^{(\mathrm{HVP}-\mathrm{B})} \leq 95.9 \times 10^{-11}, \quad \text { for } \quad 250 \mathrm{MeV} \geq M_{Q} \geq 230 \mathrm{MeV} .
$$

This result is to be compared with the phenomenological determination of this contribution which gives [23]:

$$
a_{\mu}^{(\mathrm{HVP}-\mathrm{B})}=(106.0 \pm 0.9) \times 10^{-11}
$$

We find again that, within the expected accuracy of the model, the $\mathrm{C} \chi \mathrm{QM}$ reproduces the phenomenological determination. 


\subsection{Class C: iterated HVP contributions}

This is the contribution in figure 6 induced by the quadratic term in the expansion of the photon propagator in the lowest order vertex, fully dressed by hadronic vacuum polarization corrections, i.e.

$$
D_{\alpha \beta}^{(\mathrm{HVP})}(q)=-i\left(g_{\alpha \beta}-\frac{q_{\alpha} q_{\beta}}{q^{2}}\right) \frac{1}{q^{2}} \frac{1}{1+\Pi^{(\mathrm{HVP})}\left(q^{2}\right)}-i a \frac{q_{\alpha} q_{\beta}}{q^{4}},
$$

where $\Pi^{(\mathrm{HVP})}\left(q^{2}\right)$ denotes the proper vacuum polarization self-energy contribution induced by hadrons and $a$ is a parameter reflecting the gauge freedom in the free-field propagator ( $a=1$ in the Feynman gauge). In fact, since the diagrams we are considering are gauge independent, terms proportional to $q_{\alpha} q_{\beta}$ do not contribute to their evaluation. The lowest order muon anomaly is then modified as follows:

$$
\left(\frac{\alpha}{\pi}\right) \frac{1}{2} \Rightarrow\left(\frac{\alpha}{\pi}\right) \int_{0}^{1} d x(1-x) \frac{1}{1+\Pi^{(\mathrm{HVP})}\left(\frac{-x^{2}}{1-x} m_{\mu}^{2}\right)},
$$

and the perturbation theory expansion generates a series in powers of the self-energy function $\Pi^{(\mathrm{HVP})}\left(\frac{-x^{2}}{1-x} m_{\mu}^{2}\right)$ :

$$
\frac{1}{1+\Pi^{(\mathrm{HVP})}\left(\frac{-x^{2}}{1-x} m_{\mu}^{2}\right)}=1-\Pi^{(\mathrm{HVP})}\left(\frac{-x^{2}}{1-x} m_{\mu}^{2}\right)+\left[\Pi^{(\mathrm{HVP})}\left(\frac{-x^{2}}{1-x} m_{\mu}^{2}\right)\right]^{2}+\cdots
$$

Writing a dispersion relation for each power of $\Pi^{(\mathrm{HVP})}\left(\frac{-x^{2}}{1-x} m_{\mu}^{2}\right)$ to lowest order in the electromagnetic hadronic interaction, i.e.,

$$
\Pi^{(\mathrm{HVP})}\left(\frac{-x^{2}}{1-x} m_{\mu}^{2}\right)=\int_{4 m_{\pi}^{2}}^{\infty} \frac{d t}{t} \frac{\frac{-x^{2}}{1-x} m_{\mu}^{2}}{t-\frac{-x^{2}}{1-x} m_{\mu}^{2}-i \epsilon} \frac{1}{\pi} \operatorname{Im} \Pi^{(\mathrm{HVP})}(t),
$$

results then, from the quadratic term of the expansion in eq. (3.14), in the following representation [15] for the contribution from the Feynman diagrams in figure 6

$$
a_{\mu}^{(\mathrm{HVP}-\mathrm{C})}=\int_{4 m_{\pi}^{2}}^{\infty} \frac{d t}{t} \frac{1}{\pi} \operatorname{Im} \Pi^{(\mathrm{HVP})}(t) \int_{4 m_{\pi}^{2}}^{\infty} \frac{d t^{\prime}}{t^{\prime}} \frac{1}{\pi} \operatorname{Im} \Pi^{(\mathrm{HVP})}\left(t^{\prime}\right) K\left(\frac{t}{m_{\mu}^{2}}, \frac{t^{\prime}}{m_{\mu}^{2}}\right),
$$

with

$$
K\left(\frac{t}{m_{\mu}^{2}}, \frac{t^{\prime}}{m_{\mu}^{2}}\right)=\left(\frac{\alpha}{\pi}\right) \int_{0}^{1} d x \frac{x^{4}(1-x)}{\left(x^{2}+\frac{t}{m_{\mu}^{2}}(1-x)\right)\left(x^{2}+\frac{t^{\prime}}{m_{\mu}^{2}}(1-x)\right)},
$$

a composite kernel which correlates the two spectral functions. Using the $\mathrm{C} \chi \mathrm{QM}$ spectral function in eq. (2.5) for both $\frac{1}{\pi} \operatorname{Im} \Pi^{(\mathrm{HVP})}(t)$ and $\frac{1}{\pi} \operatorname{Im} \Pi^{(\mathrm{HVP})}\left(t^{\prime}\right)$, we find a small contribution from this C-class:

$$
a_{\mu}^{(\mathrm{HVP}-\mathrm{C})}\left(M_{Q}=240 \mathrm{MeV}\right)=2.2 \times 10^{-11},
$$

with a range

$$
1.9 \times 10^{-11} \leq a_{\mu}^{(\mathrm{HVP}-\mathrm{C})} \leq 2.5 \times 10^{-11}, \quad \text { for } \quad 250 \mathrm{MeV} \geq M_{Q} \geq 230 \mathrm{MeV} .
$$


Two independent phenomenological determinations of this contribution (with errors which are likely to have been underestimated) give:

$$
a_{\mu}^{(\mathrm{HVP}-\mathrm{C})}(\text { ref. }[23])=(3.4 \pm 0.1) \times 10^{-11},
$$

and

$$
a_{\mu}^{(\mathrm{HVP}-\mathrm{C})}(\text { ref. [30] })=(3.0 \pm 0.1) \times 10^{-11} .
$$

Again, they compare reasonably well with the $\mathrm{C} \chi \mathrm{QM}$ prediction.

- Why is this contribution so small?

This is an interesting question which, to our knowledge, has not been addressed in the literature. We wish to take the opportunity to answer it here.

The main point is the following: instead of writing a dispersion relation for each power of $\Pi^{(\mathrm{HVP})}\left(q^{2}\right)$, we could have chosen to write a dispersion relation for the squared photon self-energy $\left[\Pi^{(H V P)}\left(q^{2}\right)\right]^{2}$, i.e.

$$
\left[\Pi^{(\mathrm{HVP})}\left(q^{2}\right)\right]^{2}=\int_{0}^{\infty} \frac{d t}{t} \frac{q^{2}}{t-q^{2}-i \epsilon} 2 \operatorname{Re} \Pi^{(\mathrm{HVP})}(t) \frac{1}{\pi} \operatorname{Im} \Pi^{(\mathrm{HVP})}(t) .
$$

This leads to a representation for the muon anomaly $\left(q^{2} \equiv \frac{-x^{2}}{1-x} m_{\mu}^{2}\right)$ :

$$
a_{\mu}^{(\mathrm{HVP}-\mathrm{C})}=\left(\frac{\alpha}{\pi}\right) \int_{4 m_{\pi}^{2}}^{\infty} \frac{d t}{t} \int_{0}^{1} d x \frac{x^{2}(1-x)}{x^{2}+\frac{t}{m_{\mu}^{2}}(1-x)}\left[-2 \operatorname{Re} \Pi^{(\mathrm{HVP})}(t) \frac{1}{\pi} \operatorname{Im} \Pi^{(\mathrm{HVP})}(t)\right],
$$

similar to the one we have used in eq. (3.6) for the evaluation of $a_{\mu}^{(\mathrm{HVP}-\mathrm{B})}$. Gauge invariance guarantees that the subtraction constant in the double dispersion relation

$$
\begin{aligned}
& \Pi^{(\mathrm{HVP})}\left(q^{2}\right) \times \Pi^{(\mathrm{HVP})}\left(q^{2}\right)= \\
& \quad \int_{0}^{\infty} \frac{d t}{t} \frac{q^{2}}{t-q^{2}-i \epsilon} \frac{1}{\pi} \operatorname{Im} \Pi^{(\mathrm{HVP})}(t) \times \int_{0}^{\infty} \frac{d t^{\prime}}{t^{\prime}} \frac{q^{2}}{t^{\prime}-q^{2}-i \epsilon} \frac{1}{\pi} \operatorname{Im} \Pi^{(\mathrm{HVP})}\left(t^{\prime}\right)
\end{aligned}
$$

and the one in the single dispersion relation in eq. (3.22) are the same, so that the physical electric charge corresponds to the one measured classically. In other words, gauge invariance guarantees that the physical content of the two equations (3.24) and (3.22) must be the same. Yet, algebraically, starting with the r.h.s. in eq. (3.24) and using the partial fraction decomposition:

$$
\frac{1}{t-q^{2}-i \epsilon} \frac{1}{t^{\prime}-q^{2}-i \epsilon}=\frac{1}{t-q^{2}-i \epsilon} \frac{1}{t^{\prime}-t}+\frac{1}{t^{\prime}-q^{2}-i \epsilon} \frac{1}{t-t^{\prime}}
$$

one gets the following relation:

$$
\Pi^{(\mathrm{HVP})}\left(q^{2}\right) \times \Pi^{(\mathrm{HVP})}\left(q^{2}\right)=\left[\Pi^{(\mathrm{HVP})}\left(q^{2}\right)\right]^{2}-q^{2} \int_{0}^{\infty} \frac{d t}{t^{2}} 2 \operatorname{Re} \Pi^{(\mathrm{HVP})}(t) \frac{1}{\pi} \operatorname{Im} \Pi^{(\mathrm{HVP})}(t) .
$$


Obviously, the only way to preserve the identity $\Pi^{(\mathrm{HVP})}\left(q^{2}\right) \times \Pi^{(\mathrm{HVP})}\left(q^{2}\right)=$ $\left[\Pi^{(\mathrm{HVP})}\left(q^{2}\right)\right]^{2}$ is that

$$
\int_{0}^{\infty} \frac{d t}{t^{2}} 2 \operatorname{Re} \Pi^{(\mathrm{HVP})}(t) \frac{1}{\pi} \operatorname{Im} \Pi^{(\mathrm{HVP})}(t)=0
$$

which is a highly non trivial constraint! ${ }^{4}$ It is this constraint which answers the question of why $a_{\mu}^{(\mathrm{HVP}-\mathrm{C})}$ turns out to be so small. Indeed, it implies that the a priori leading term of $\mathcal{O}\left(m_{\mu}^{2}\right)$ in an expansion in powers of $m_{\mu}^{2}$ in the r.h.s. of eq. (3.23), contrary to what happens with the lowest order hadronic vacuum polarization contribution in eq. (2.1) where it provides the dominant contribution, is not there in the double hadronic vacuum polarization contribution. The leading term in a $m_{\mu^{-}}^{2}$ expansion for $a_{\mu}^{(\mathrm{HVP}-\mathrm{C})}$ must be $\mathcal{O}\left(m_{\mu}^{4}\right)$ at least. In fact, a detailed analysis shows that it is $\mathcal{O}\left[\frac{m_{\mu}^{4}}{M_{\mathrm{H}}^{4}} \log \left(\frac{M_{\mathrm{H}}}{m_{\mu}}\right)\right]$, with $M_{\mathrm{H}}$ a hadronic scale which in the $\mathrm{C} \chi \mathrm{QM}$ is $M_{Q}$ of course. This is the reason why the double hadronic vacuum polarization contribution to the muon anomaly is so small and, as we have shown, this is a model independent statement.

\section{- Comment on radiative corrections}

Hadronic vacuum polarization generates part of the radiative corrections to the total $e^{+} e^{-}$annihilation bare cross-section into hadrons. In fact this correction leads to the following modification of the bare cross-section

$$
\begin{aligned}
\sigma(t)_{e^{+} e^{-} \rightarrow \text { hadrons }} & =\frac{4 \pi^{2} \alpha}{t} \frac{1}{\pi} \operatorname{Im} \Pi^{(\mathrm{HVP})}(t) \\
& \Rightarrow \frac{4 \pi^{2} \alpha}{t}\left[1-2 \operatorname{Re} \Pi^{(\mathrm{HVP})}(t)\right] \frac{1}{\pi} \operatorname{Im} \Pi^{(\mathrm{HVP})}(t),
\end{aligned}
$$

which corresponds to the modification

$$
\frac{1}{\pi} \operatorname{Im} \Pi^{(\mathrm{HVP})}(t) \Rightarrow\left[1-2 \operatorname{Re} \Pi^{(\mathrm{HVP})}(t)\right] \frac{1}{\pi} \operatorname{Im} \Pi^{(\mathrm{HVP})}(t),
$$

and leads, precisely, to the muon anomaly contribution given in eq. (3.23). In other words, if in the lowest order expression for the muon anomaly one inserts the bare total $e^{+} e^{-}$annihilation cross-section into hadrons, we are indeed calculating the lowest order contribution $a_{\mu}^{(\mathrm{HVP})}$ in eq. (2.1). This implies that the appropriate radiative corrections to the physical cross-section have been made including the correction due to hadronic vacuum polarization. The alternative is to leave the physical cross-section uncorrected for hadronic vacuum polarization, in which case, when inserted in the lowest order expression, one is then calculating: $a_{\mu}^{(\mathrm{HVP})}+a_{\mu}^{(\mathrm{HVP}-\mathrm{C})}$. Then, obviously, one should not add an extra independent evaluation of $a_{\mu}^{(\mathrm{HVP}-\mathrm{C})}$.

The warning here, specially for theorists, is that in using experimental hadronic cross-sections to compute hadronic vacuum polarization contributions to the muon

\footnotetext{
${ }^{4} \mathrm{In}$ QED, one can easily check this constraint in perturbation theory.
} 


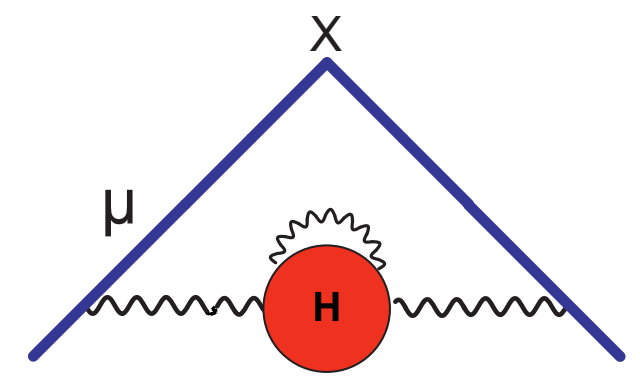

Figure 7. Feynman diagrams corresponding to the Class D contribution to the muon anomaly.

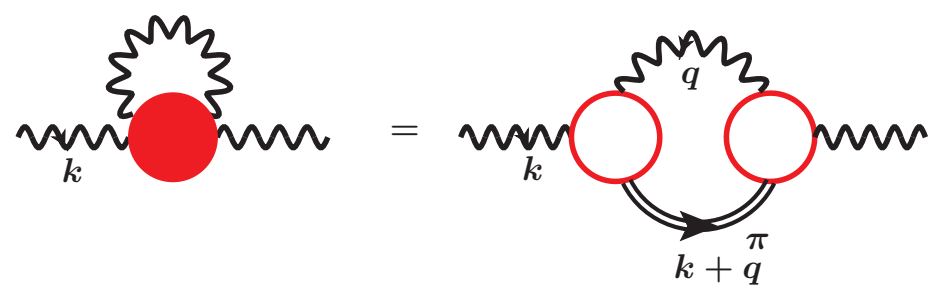

(a)

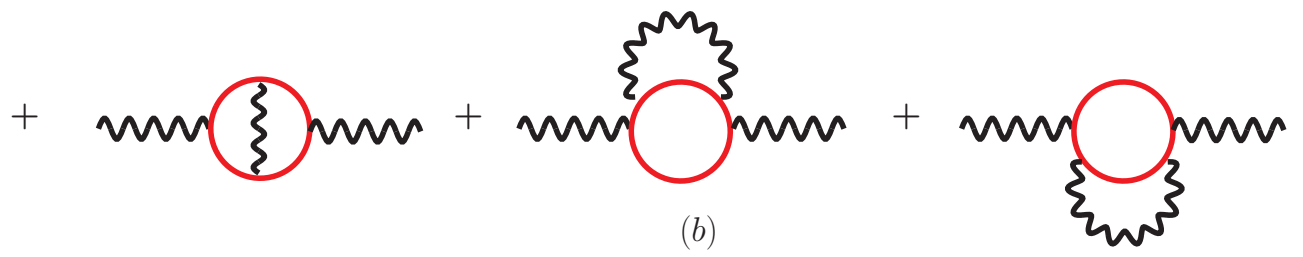

Figure 8. Feynman diagrams which contribute to the photon self-energy to $\mathcal{O}\left(\alpha^{2}\right)$ in the $\mathrm{C} \chi \mathrm{QM}$.

anomaly, one should be very careful to know exactly what these cross-sections correspond to. Often the data which is used corresponds to different experiments which complicates even further the issue.

Another warning, this one for experimental physicists, concerns the dynamical constraint given in eq. (3.27). In doing hadronic vacuum polarization corrections to the total cross-section numerically (e.g. involving an iterative procedure, as mentioned in some of the experimental papers) one should be careful to check that this constraint, which involves rather subtle cancellations, is indeed satisfied.

\subsection{Class D: contributions with HVP corrections at $\mathcal{O}(\alpha)$}

In the $\mathrm{C} \chi \mathrm{QM}$ there are two types of contributions to this class: the $\pi^{0} \gamma$ exchange and the constituent quark loop with a virtual photon insertion. They correspond to the photon propagator content illustrated in figure 8. Corresponding to these two subclasses we shall write

$$
a_{\mu}^{(\mathrm{HVP}-\mathrm{D})}=a_{\mu}^{\left(\pi^{0} \gamma\right)}+a_{\mu}^{(Q, \alpha)}
$$

and discuss separately the two contributions. They are both leading in the Large- $\mathrm{N}_{\mathrm{c}}$ limit. 


\subsubsection{Contribution from the $\pi^{0} \gamma$ intermediate state}

Here it is convenient to use the representation (see page 231 in ref. [24] and ref. [25]: ${ }^{5}$ )

$$
a_{\mu}^{\left(\pi^{0} \gamma\right)}=\frac{\alpha}{\pi} \int_{0}^{1} d z(1-z)\left[-\Pi^{\left(\pi^{0} \gamma\right)}\left(-\frac{z^{2}}{1-z} m_{\mu}^{2}\right)\right]
$$

where $\Pi^{\left(\pi^{0} \gamma\right)}\left(k^{2}\right)$ denotes the renormalized photon self-energy from the $\pi^{0} \gamma$ contribution and the integration is over the value of the self-energy in the euclidean. In fact, we find that a better representation, which avoids renormalization issues, is the one in terms of the Adler function [26]. ${ }^{6}$ Using integration by parts in eq. (3.31) with $(1-z)=-\frac{1}{2} \frac{d}{d z}(1-z)^{2}$ and the fact that $\Pi(0)=0$, one finds

$$
a_{\mu}^{\left(\pi^{0} \gamma\right)}=\frac{\alpha}{2 \pi} \int_{0}^{1} \frac{d z}{z}(1-z)(2-z) \mathcal{A}^{\left(\pi^{0} \gamma\right)}\left(\frac{z^{2}}{1-z} m_{\mu}^{2}\right)
$$

with $\mathcal{A}^{\left(\pi^{0} \gamma\right)}\left(Q^{2}\right)$ the Adler function $\left(Q^{2}=-k^{2}\right)$

$$
\mathcal{A}^{\left(\pi^{0} \gamma\right)}\left(Q^{2}\right)=-Q^{2} \frac{d \Pi^{\left(\pi^{0} \gamma\right)}\left(k^{2}\right)}{d Q^{2}}, \quad Q^{2} \equiv \frac{z^{2}}{1-z} m_{\mu}^{2} .
$$

In the $\mathrm{C} \chi \mathrm{QM}$, the $\pi^{0} \gamma \gamma$ three-point function at each vertex in the first diagram of figure 10(a) can be expressed in terms of the following parametric representation:

$$
\begin{aligned}
\mathcal{F}_{\pi^{0 *} \gamma^{*} \gamma^{*}}^{(\mathrm{QM})} & \left((k+q)^{2}, k^{2}, q^{2}\right)=-i e^{2} \frac{N_{c}}{12 \pi^{2} f_{\pi}} \times \\
& \times \int_{0}^{1} d x x \int_{0}^{1} d y \frac{2 M_{Q}^{2}}{M_{Q}^{2}-x(1-x)(1-y) k^{2}-x^{2} y(1-y)(k+q)^{2}-x y(1-x) q^{2}-i \epsilon} .
\end{aligned}
$$

Here, the constituent quark mass $M_{Q}$ acts as an UV-regulator of the $\pi^{0} \gamma$ contribution to the muon anomaly. In the limit $M_{Q} \rightarrow \infty$ this form factor reduces to the $\pi^{0} \gamma \gamma$ Adler, Bell-Jackiw point-like coupling (ABJ):

$$
\mathcal{F}_{\pi^{0} \gamma^{*} \gamma^{*}}^{(\chi \mathrm{QM})}\left((k+q)^{2}, k^{2}, q^{2}\right) \underset{M_{Q} \rightarrow \infty}{\longrightarrow}-i e^{2} \frac{N_{c}}{12 \pi^{2} f_{\pi}},
$$

and in this limit, the contribution to the muon anomaly becomes UV-divergent. Using dimensional regularization and the $\overline{\mathrm{MS}}$-renormalization scheme, the result in this limit, with $m_{\mu}^{2} \ll m_{\pi}^{2}$ for further simplification, and $\mu$ the renormalization scale, is

$$
a_{\mu}^{\left(\pi^{0} \gamma\right)}(A B J)=\left(\frac{\alpha}{\pi}\right)^{3} \frac{m_{\mu}^{2}}{16 \pi^{2} f_{\pi}^{2}} \frac{N_{c}^{2}}{162}\left\{\log \frac{\mu^{2}}{m_{\pi}^{2}}+\frac{5}{6}+\mathcal{O}\left[\frac{m_{\mu}^{2}}{m_{\pi}^{2}} \log \frac{m_{\pi}^{2}}{m_{\mu}^{2}}\right]\right\} .
$$

In particular, for $\mu=M_{\rho}$, one finds

$$
a_{\mu}^{\left(\pi^{0} \gamma\right)}(A B J)_{\mu=M_{\rho}}=2.5 \times 10^{-11} ;
$$

\footnotetext{
${ }^{5}$ This is a representation which is now often used by our lattice QCD colleagues to compute the hadronic vacuum polarization contribution.

${ }^{6}$ Fred Jegerlehner has often advocated also the advantages of this representation.
} 
a result which, within a $30 \%$ error, is consistent with the one in ref. [27]:

$$
a_{\mu}^{(\pi \gamma)} \simeq 3.7 \times 10^{-11}
$$

obtained with Vector Meson Dominance like form factors:

$$
\mathcal{F}_{\pi^{0 *} \gamma^{*} \gamma^{*}}^{(\mathrm{VMD})}\left((k+q)^{2}, k^{2}, q^{2}\right)=-i e^{2} \frac{N_{c}}{12 \pi^{2} f_{\pi}} \frac{M_{\rho}^{2}}{M_{\rho}^{2}-k^{2}} \frac{M_{\rho}^{2}}{M_{\rho}^{2}-q^{2}} .
$$

The Feynman parameterization of the full $\pi^{0} \gamma$ Adler function in the $\mathrm{C} \chi \mathrm{QM}$, using the form factor expression in eq. (3.34), results in the following representation:

$$
\begin{aligned}
\mathcal{A}^{\left(\pi^{0} \gamma\right)}\left(Q^{2}\right)= & \left(\frac{\alpha}{\pi}\right)^{2} N_{c}^{2} \frac{Q^{2}}{16 \pi^{2} f_{\pi}^{2}} \frac{4}{9} \int_{0}^{1} d u u^{2} \int_{0}^{1} d v v \int_{0}^{1} d w \\
& \times \int_{0}^{1} d x x \int_{0}^{1} d y \int_{0}^{1} d x^{\prime} x^{\prime} \int_{0}^{1} d y^{\prime} \frac{1}{x y(1-x y) x^{\prime} y^{\prime}\left(1-x^{\prime} y^{\prime}\right)} \times \\
& \times\left\{\frac{(1-y)[1-x(1-y)]}{y(1-x y)} u(1-v)+\frac{\left(1-y^{\prime}\right)\left[1-x^{\prime}\left(1-y^{\prime}\right)\right]}{y^{\prime}\left(1-x^{\prime} y^{\prime}\right)}(1-u)\right. \\
& \left.\quad+u v(1-w)-\left[u v(1-w)+u(1-v) \delta+(1-u) \delta^{\prime}\right]^{2}\right\}\left(\frac{M_{Q}^{2}}{\mathcal{R}^{2}}\right)^{2},
\end{aligned}
$$

where

$$
\begin{aligned}
\mathcal{R}^{2}= & Q^{2}\left\{\frac{(1-y)[1-x(1-y)]}{y(1-x y)} u(1-v)+\frac{\left(1-y^{\prime}\right)\left[1-x^{\prime}\left(1-y^{\prime}\right)\right]}{y^{\prime}\left(1-x^{\prime} y^{\prime}\right)}(1-u)\right. \\
& \left.+u v(1-w)-\left[u v(1-w)+u(1-v) \delta+(1-u) \delta^{\prime}\right]^{2}\right\} \\
& +M_{Q}^{2}\left[\frac{u(1-v)}{x y(1-x y)}+\frac{1-u}{x^{\prime} y^{\prime}\left(1-x^{\prime} y^{\prime}\right)}\right]+m_{\pi}^{2} u v(1-w) .
\end{aligned}
$$

and

$$
\delta=\frac{x(1-y)}{1-x y} \quad \text { and } \quad \delta^{\prime}=\frac{x^{\prime}\left(1-y^{\prime}\right)}{1-x^{\prime} y^{\prime}} .
$$

Performing the integration over the seven Feynman parameters numerically we find the following results:

$$
a_{\mu}^{(\pi \gamma)}\left(M_{Q}=240 \mathrm{MeV}\right)=2.17 \times 10^{-11},
$$

with a range

$$
2.10 \times 10^{-11} \leq a_{\mu}^{(\pi \gamma)} \leq 2.18 \times 10^{-11}, \quad \text { for } \quad 230 \mathrm{MeV} \leq M_{Q} \leq 250 \mathrm{MeV},
$$

consistent with the estimate in eq. (3.37).

We observe, however, that these results turn out to be an order of magnitude smaller than the phenomenological contribution quoted in the table 2 of ref. [4] (see also ref. [23]):

$$
a_{\mu}^{(\pi \gamma)}=(44.2 \pm 1.9) \times 10^{-11},
$$

which uses as input the measured $\sigma\left(e^{+} e^{-} \rightarrow \pi^{0} \gamma\right)$ cross section in the energy interval $0.60<\sqrt{s}<1.03 \mathrm{GeV}[28]$. 


\section{- Why this discrepancy?}

In order to understand better the underlying physics let us use instead the representation for $a_{\mu}^{(\pi \gamma)}$ in terms of the spectral function $\frac{1}{\pi} \operatorname{Im} \Pi^{(\pi \gamma)}(t)$ i.e.,

$$
a_{\mu}^{(\pi \gamma)}=\int_{m_{\pi}^{2}}^{\infty} \frac{d t}{t} K\left(\frac{t}{m_{\mu}^{2}}\right) \frac{1}{\pi} \operatorname{Im} \Pi^{(\pi \gamma)}(t) .
$$

The phenomenological determinations of $a_{\mu}^{(\pi \gamma)}$ in refs. $[4,23]$ implicitly assume that $\frac{1}{\pi} \operatorname{Im} \Pi^{(\pi \gamma)}(t)$ is completely saturated by the $\pi^{0} \gamma$ intermediate state. Notice however that in the $\mathrm{C} \chi \mathrm{QM}$ there are other intermediate states which also contribute to $\frac{1}{\pi} \operatorname{Im} \Pi^{(\pi \gamma)}(t)$; they correspond to the $Q \bar{Q}, Q \bar{Q} \gamma$ and $Q \bar{Q} \pi$ discontinuities in the diagram (a) of figure 8 . These discontinuities are automatically included in the calculation of $a_{\mu}^{\left(\pi^{0} \gamma\right)}$ which uses the euclidean representation in eq. (3.32). In order to compare the $\mathrm{C} \chi \mathrm{QM}$ determination to the phenomenological ones, let us then restrict $\frac{1}{\pi} \operatorname{Im} \Pi^{(\pi \gamma)}(t)$ to the contribution from the on-shell $\pi^{0} \gamma$ intermediate state only. Then

$$
\left.\frac{1}{\pi} \operatorname{Im} \Pi^{(\pi \gamma)}(t)\right|_{\pi \gamma}=\left(\frac{\alpha}{\pi}\right)^{2} \frac{N_{c}^{2}}{16 \pi^{2} f_{\pi}^{2}} \frac{1}{54} t\left(1-\frac{m_{\pi}^{2}}{t}\right)^{3}\left|\tilde{\mathcal{F}}_{\pi^{0} \gamma^{*} \gamma}^{(\chi \mathrm{QM})}\left(m_{\pi}^{2}, t, 0\right)\right|^{2},
$$

with

$$
\tilde{\mathcal{F}}_{\pi^{0} \gamma^{*} \gamma}^{(\chi \mathrm{QM})}\left(m_{\pi}^{2}, t, 0\right)=\int_{0}^{1} d x x \int_{0}^{1} d y \frac{2 M_{Q}^{2}}{M_{Q}^{2}-x(1-x)(1-y) t-x^{2} y(1-y) m_{\pi}^{2}-i \epsilon} .
$$

This form factor has an imaginary part which, for $m_{\pi} \leq 2 M_{Q}$, is:

$$
\frac{1}{\pi} \operatorname{Im} \tilde{\mathcal{F}}_{\pi^{0} \gamma^{*} \gamma}^{(\chi Q M)}\left(m_{\pi}^{2}, t, 0\right)=\frac{4 M_{Q}^{2}}{t-m_{\pi}^{2}} \log \frac{\sqrt{t}}{2 M_{Q}}\left(1+\sqrt{1-\frac{4 M_{Q}^{2}}{t}}\right) \theta\left(t-4 M_{Q}^{2}\right) .
$$

and a real part:

$$
\begin{aligned}
\operatorname{Re} \tilde{\mathcal{F}}_{\phi \gamma^{*} \gamma}^{\left(\pi^{0} Q M\right)}\left(m_{\pi}^{2}, t, 0\right)= & \frac{4 M_{Q}^{2}}{t-m_{\pi}^{2}}\left[\arctan ^{2}\left(\frac{1}{\sqrt{\frac{4 M_{Q}^{2}}{m_{\pi}^{2}}-1}}\right)\right. \\
& +\left\{\begin{array}{l}
-\arctan ^{2}\left(\frac{1}{\sqrt{\frac{4 M_{Q}^{2}}{t}-1}}\right), \quad m_{\pi}^{2} \leq t \leq 4 M_{Q}^{2} \\
\ln ^{2} \frac{\sqrt{t}}{2 M_{Q}}\left(1+\sqrt{1-\frac{4 M_{Q}^{2}}{t}}\right)-\frac{\pi^{2}}{4}, \quad t \geq 4 M_{Q}^{2}
\end{array}\right] .
\end{aligned}
$$

The shape of the spectral function in eq. (3.47), in units of $\left(\frac{\alpha}{\pi}\right)^{2}$ and for the value $M_{Q}=240 \mathrm{MeV}$, is shown in figure 9 .

The result of the integral in eq. (3.47) with the spectral function plotted in figure 9 is then

$$
a_{\mu}^{(\pi \gamma)}(\text { eq. }(3.46))=3.0 \times 10^{-11} \text { for } \quad M_{Q}=240 \mathrm{MeV},
$$




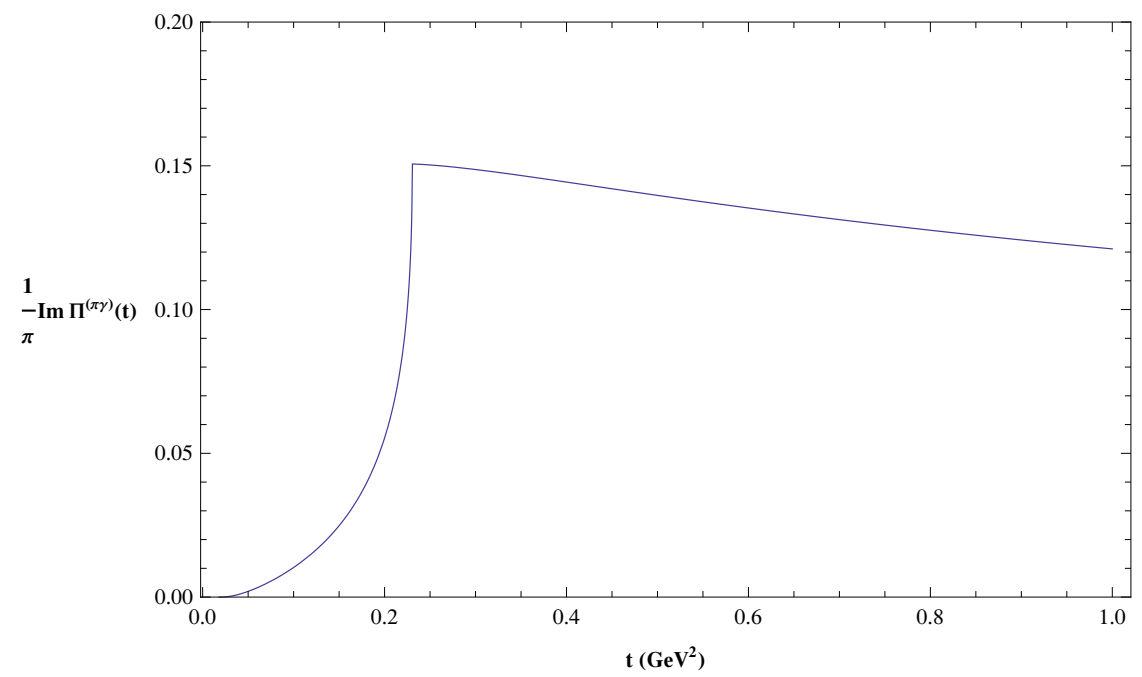

Figure 9. The spectral function in eq. (3.47), in units of $\left(\frac{\alpha}{\pi}\right)^{2}$, for $M_{Q}=240 \mathrm{MeV}$.

a result which is slightly higher than the full $\mathrm{C} \chi \mathrm{QM}$ contribution in eq. (3.43) but still well below the phenomenological determinations [4, 23].

Let us then try to simplify the phenomenological determination as much as possible to see where the big contribution comes from. For that, it will be sufficient to approximate eq. (3.46) as follows:

$$
a_{\mu}^{(\pi \gamma)} \sim\left(\frac{\alpha}{\pi}\right) \int_{m_{\pi}^{2}}^{\infty} \frac{d t}{t} \frac{1}{3} \frac{m_{\mu}^{2}}{t} \frac{1}{\pi} \operatorname{Im} \Pi^{(\pi \gamma)}(t)
$$

and use a narrow width expression for the spectral function, which as we shall soon see, is dominated by the $\omega$ contribution. This results in the simple formula:

$$
a_{\mu}^{(\pi \gamma)} \sim \frac{1}{3} \frac{m_{\mu}^{2}}{M_{\omega}^{2}} \frac{4}{\pi} \frac{\Gamma\left(\omega \rightarrow e^{+} e^{-}\right)}{M_{\omega}} \frac{\Gamma\left(\omega \rightarrow \pi^{0} \gamma\right)}{\Gamma_{\omega}}=53 \times 10^{-11}
$$

which reproduces, in order of magnitude, the phenomenological estimates. We can, therefore, see that the big number comes from the large experimental value of the branching ratio

$$
\frac{\Gamma\left(\omega \rightarrow \pi^{0} \gamma\right)}{\Gamma_{\omega}} \simeq 8 \times 10^{-2} .
$$

Notice that in the case of the $\rho$ contribution the corresponding branching ratio is much smaller:

$$
\frac{\Gamma\left(\rho \rightarrow \pi^{0} \gamma\right)}{\Gamma_{\rho}} \simeq 6 \times 10^{-4}
$$

It is the large branching ratio in eq. (3.54) which the $\mathrm{C} \chi \mathrm{QM}$ fails to reproduce!

Phenomenologically, the large branching ratio $\frac{\Gamma\left(\omega \rightarrow \pi^{0} \gamma\right)}{\Gamma\left(\rho \rightarrow \pi^{0} \gamma\right)}$ is due to the $\omega-\phi$ mixing and the fact that the $\phi$ is an almost pure $s \bar{s}$ state. ${ }^{7}$ By construction, the $\mathrm{C} \chi \mathrm{QM}$ form

\footnotetext{
${ }^{7}$ We thank Marc Knecht for reminding us of this.
} 
factor is $\mathrm{SU}(3)$ invariant and, therefore, like any model which is $\mathrm{SU}(3)$ invariant, fails to reproduce this phenomenological fact.

We are aware of the fact that in the $\mathrm{C} \chi \mathrm{QM}$ there are also further contributions of the $\pi^{0} \gamma$ subclass: those from the $\eta_{8} \gamma$ and $\eta_{0} \gamma$ intermediate states. We refrain from discussing them because their comparison with their corresponding phenomenological determinations requires issues like $\eta_{8}-\eta_{0}$ mixing as well as the question of the $\eta^{\prime}$ mass in Large- $\mathrm{N}_{\mathrm{c}}$ which are beyond the scope of the model we are discussing.

\subsubsection{Contribution from the quark loop to $\mathcal{O}(\alpha)$}

This contribution is given by the following integral representation:

$$
a_{\mu}^{(Q, \alpha)}=\int_{4 M_{Q}^{2}}^{\infty} \frac{d t}{t} K\left(t / m_{\mu}^{2}\right) \frac{1}{\pi} \operatorname{Im} \Pi^{(Q, \alpha)}(t)
$$

where

$$
\frac{1}{\pi} \operatorname{Im} \Pi^{(Q, \alpha)}(t)=\left(\frac{\alpha}{\pi}\right)^{2} N_{c}\left[\left(\frac{2}{3}\right)^{4}+\left(-\frac{1}{3}\right)^{4}+\left(-\frac{1}{3}\right)^{4}\right] \rho_{\mathrm{KS}}(t),
$$

with $\rho_{\mathrm{KS}}(t)$ given in eq. (2.6). As $M_{Q} \rightarrow \infty$ it decouples with a leading behaviour:

$$
\begin{aligned}
a_{\mu}^{(Q, \alpha)} & \simeq\left(\frac{\alpha}{\pi}\right)^{3} N_{c} \frac{2}{9} \frac{1}{3} \frac{m_{\mu}^{2}}{M_{Q}^{2}}\left(\int_{4 M_{Q}^{2}}^{\infty} \frac{d t}{t} \frac{M_{Q}^{2}}{t} \rho_{\mathrm{KS}}(t)=\frac{41}{162}\right) \\
& =13.7 \times 10^{-11} \text { for } \quad M_{Q}=240 \mathrm{MeV} .
\end{aligned}
$$

The full numerical evaluation gives

$$
a_{\mu}^{(Q, \alpha)}\left(M_{Q}=240 \mathrm{MeV}\right)=11.4 \times 10^{-11},
$$

with a range

$$
10.6 \times 10^{-11} \leq a_{\mu}^{(Q, \alpha)} \leq 12.3 \times 10^{-11}, \quad \text { for } \quad 250 \mathrm{MeV} \geq M_{Q} \geq 230 \mathrm{MeV} .
$$

Altogether we find that, although the $\pi^{0} \gamma$ exchange contribution increases logarithmically as a function of $M_{Q}$, while the quark loop decouples as an inverse power of $M_{Q}^{2}$, their ratio for $M_{Q}^{2}$ large goes as

$$
\left.\frac{a_{\mu}^{(\pi \gamma)}}{a_{\mu}^{(Q, \alpha)}}\right|_{M_{Q} \rightarrow \infty} \sim \mathrm{N}_{\mathrm{c}} \frac{M_{Q}^{2}}{16 \pi^{2} f_{\pi}^{2}} \frac{27}{82} \log \frac{M_{Q}^{2}}{m_{\pi}^{2}}
$$

and, therefore, for values of the constituent quark mass in the range $250 \mathrm{MeV} \geq M_{Q} \geq$ $230 \mathrm{MeV}$, it is the quark loop contribution which still dominates.

The total sum of the two contributions of Class $\mathbf{D}$ in the $\mathrm{C} \chi \mathrm{QM}$ is then:

$$
a_{\mu}^{(\mathrm{HVP}-\mathrm{D})}\left(M_{Q}=240 \mathrm{MeV}\right)=13.6 \times 10^{-11},
$$




\begin{tabular}{|c|c|}
\hline Class & Result in $10^{-11}$ units \\
\hline A & $-171 \pm 10$ \\
B & $89 \pm 7$ \\
C & $2.2 \pm 0.3$ \\
D $(\pi \gamma)$ & $2.2 \pm 0.1$ \\
D (Q-loop $)$ & $13.5 \pm 0.5$ \\
\hline Total & $-64 \pm 12$ \\
\hline
\end{tabular}

Table 1. Results for the HVP contributions of $\mathcal{O}\left(\frac{\alpha}{\pi}\right)^{3}$ in the C $\chi$ QM.

with a range for this total

$$
12.8 \times 10^{-11} \leq a_{\mu}^{(\mathrm{HVP}-\mathrm{D})} \leq 14.4 \times 10^{-11}, \quad \text { for } \quad 250 \mathrm{MeV} \geq M_{Q} \geq 230 \mathrm{MeV} .
$$

Except for the $\pi^{0} \gamma$ contribution, it is difficult to compare the overall $\mathrm{C} \chi \mathrm{QM}$ prediction for the Class D contributions with the phenomenological estimates. The reason is that, a priori, when inserting a physical observable to evaluate the diagram in figure 7 one needs two types of contributions: the one from the cross section $\sigma\left(e^{+} e^{-} \rightarrow\right.$ Hadrons $\left.+\gamma\right)$ and the one from the interference of the amplitude $e^{+} e^{-} \rightarrow$ Hadrons with the same amplitude where a virtual photon has been emitted and reabsorbed. In fact, individually, these two contributions are infrared divergent, which complicates things even more. This is a place where it would be interesting to see if lattice QCD can eventually make an estimate of these Class D contributions which, so far, remain poorly known phenomenologically.

Table 1 gives a summary of the results for the four classes of contributions discussed here and evaluated within the framework of the $\mathrm{C} \chi \mathrm{QM}$, with a total

$$
a_{\mu}^{[\mathrm{HVP}-(\mathrm{A}, \mathrm{B}, \mathrm{C}, \mathrm{D})]}=(-64 \pm 12) \times 10^{-11} .
$$

This $\mathrm{C} \chi \mathrm{QM}$ result is to be compared with the number quoted in the latest evaluation in ref. [29] for this contribution which, however, does not include the important contributions from the $\pi^{0} \gamma$ and $\eta \gamma$ intermediate states already incorporated in the lowest order HVP contribution:

$$
a_{\mu}^{[\mathrm{HVP}-\text { nextorder }]}\left(e^{+} e^{-}\right)=\left(-98.4 \pm 0.6_{\exp } \pm 0.4_{\mathrm{rad}}\right) \times 10^{-11} .
$$

Again, except for the $\pi^{0} \gamma$ issue already discussed, the agreement within the errors of the model is quite reasonable.

\section{Hadronic light-by-light scattering contributions}

The standard representation of the contribution to the muon anomaly from the hadronic light-by-light scattering shown in figure 10 is given by the integral [31]:

$$
\begin{aligned}
a_{\mu}^{(\mathrm{HLbyL})}= & \frac{-i e^{6}}{48 m_{\mu}} \int \frac{d^{4} q_{1}}{(2 \pi)^{4}} \int \frac{d^{4} q_{2}}{(2 \pi)^{4}} \frac{1}{q_{1}^{2} q_{2}^{2}\left(q_{1}+q_{2}\right)^{2}}\left[\frac{\partial}{\partial q^{\mu}} \Pi_{\lambda \nu \rho \sigma}^{(\mathrm{H})}\left(q, q_{1}, q_{3}, q_{2}\right)\right]_{q=0} \\
& \times \operatorname{tr}\left\{\left(\not p+m_{\mu}\right)\left[\gamma^{\mu}, \gamma^{\lambda}\right]\left(\not p+m_{\mu}\right) \gamma^{\nu} \frac{1}{\not p+\not q_{2}-m_{\mu}} \gamma^{\rho} \frac{1}{\left.\not p-\not q_{1}-m_{\mu}\right)} \gamma^{\sigma}\right\},
\end{aligned}
$$




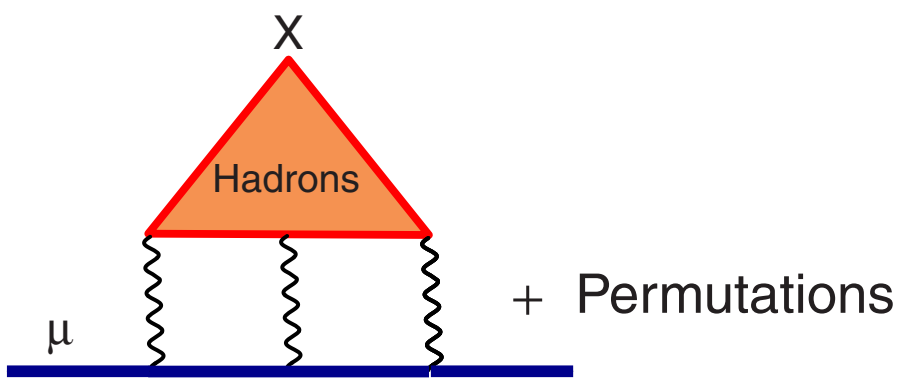

Figure 10. Hadronic light-by-light scattering contribution to the muon anomaly.

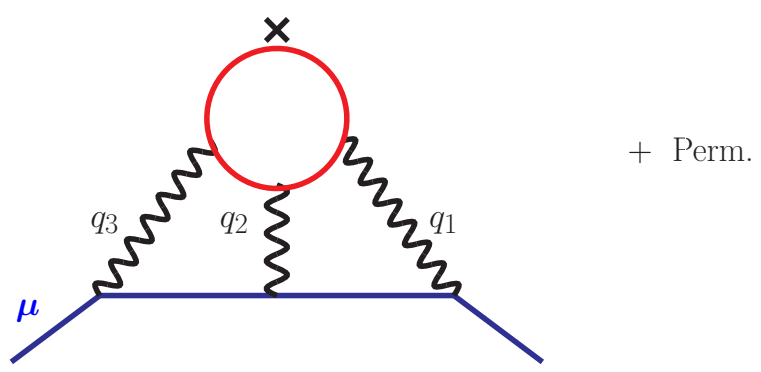

Figure 11. Constituent quark loop contribution to the muon anomaly in the C $\chi \mathrm{QM}$.
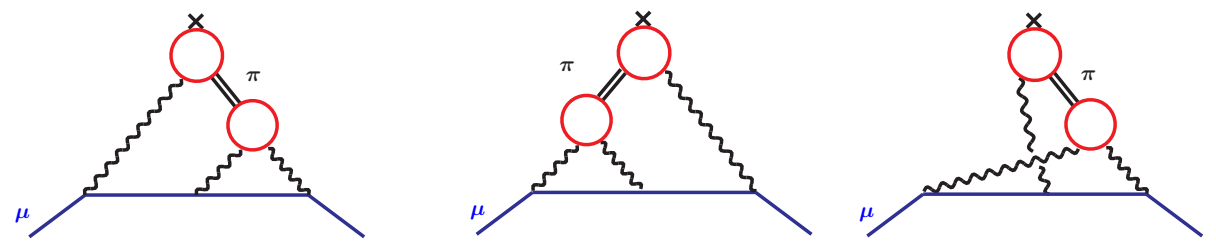

Figure 12. Goldstone exchange contribution to the muon anomaly in the $\mathrm{C} \chi \mathrm{QM}$.

where $\Pi_{\mu \nu \rho \sigma}^{(\mathrm{H})}\left(q, q_{1}, q_{3}, q_{2}\right)$, with $q=p_{2}-p_{1}=-q_{1}-q_{2}-q_{3}$, denotes the off-shell photonphoton scattering amplitude induced by hadrons,

$$
\begin{aligned}
\Pi_{\mu \nu \rho \sigma}^{(\mathrm{H})}\left(q, q_{1}, q_{3}, q_{2}\right)=\int d^{4} x_{1} \int d^{4} x_{2} \int d^{4} x_{3} & \exp \left[-i\left(q_{1} \cdot x_{1}+q_{2} \cdot x_{2}+q_{3} \cdot x_{3}\right)\right] \times \\
& \times\left\langle 0\left|T\left\{J_{\mu}(0), J_{\nu}\left(x_{1}\right), J_{\rho}\left(x_{2}\right), J_{\sigma}\left(x_{3}\right)\right\}\right| 0\right\rangle,
\end{aligned}
$$

and $J_{\mu}(x)=\sum_{q} Q_{q} \bar{q}(x) \gamma_{\mu} q(x)$ is the Standard Model electromagnetic hadronic current where, for the light quarks, $Q_{q}=\operatorname{diag}(2 / 3,-1 / 3,-1 / 3)$.

In the $\mathrm{C} \chi \mathrm{QM}$ there are two types of contributions: the Constituent Quark Loop (CQL) contribution shown in figure 11 and the Goldstone Exchange Contribution shown in figure 12 with constituent quark loops at each vertex. We shall consider these two types of contributions, both leading in the $1 / \mathrm{N}_{\mathrm{c}^{-}}$expansion, separately. 


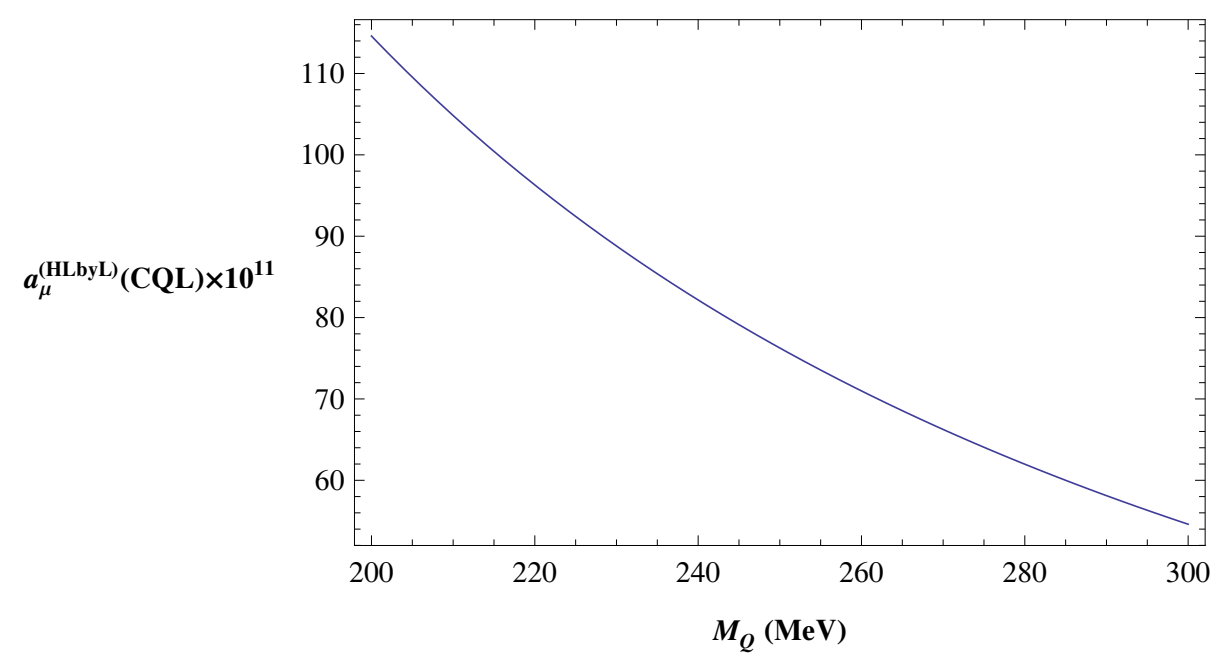

Figure 13. $a_{\mu}^{\mathrm{HLbyL}}(\mathrm{CQL})$ in eq. (4.3) in $10^{-11}$ units.

\subsection{Class A: the constituent quark loop contribution}

This contribution can be obtained from the QED analytic calculation of Laporta and Remmidi [32] with the result

$$
\begin{aligned}
a_{\mu}^{\mathrm{HLbyL}}(\mathrm{CQL})=\left(\frac{\alpha}{\pi}\right)^{3} & N_{c}\left(\sum_{q=u, d, s} Q_{q}^{4}\right)\left\{\left[\frac{3}{2} \zeta(3)-\frac{19}{16}\right] \frac{m_{\mu}^{2}}{M_{Q}^{2}}\right. \\
& -\frac{m_{\mu}^{4}}{M_{Q}^{4}}\left[\frac{161}{3240} \log ^{2} \frac{M_{Q}^{2}}{m_{\mu}^{2}}+\frac{16189}{97200} \log \frac{M_{Q}^{2}}{m_{\mu}^{2}}-\frac{13}{18} \zeta(3)+\frac{161}{1620} \frac{\pi^{2}}{6}+\frac{831931}{972000}\right] \\
& \left.+\mathcal{O}\left(\frac{m_{\mu}^{6}}{M_{Q}^{6}} \log ^{2} \frac{m_{\mu}^{2}}{M_{Q}^{2}}\right)\right\}
\end{aligned}
$$

A plot of this contribution versus $M_{Q}$ is shown in figure 13 . We find

$$
a_{\mu}^{\mathrm{HLbyL}}(\mathrm{CQL})=82.2 \times 10^{-11} \text { at } M_{Q}=240 \mathrm{MeV},
$$

with a range

$$
76.3 \times 10^{-11} \leq a_{\mu}^{\mathrm{HLbyL}}(\mathrm{CQL}) \leq 88.8 \times 10^{-11} \text { for } \quad 250 \mathrm{MeV} \geq M_{Q} \geq 230 \mathrm{MeV}
$$

The gluonic corrections of $\mathcal{O}\left(\frac{\alpha_{\mathrm{S}}}{\pi}\right)$ to the leading term in eq. (4.3) have been recently calculated in ref. [33] and found to be rather small. 


\subsection{Class B: the $\pi^{0}$ exchange contribution}

The expression for this contribution in terms of the vertex form factors $\mathcal{F}_{\pi^{0 *}} \gamma^{*} \gamma^{*}$ and $\mathcal{F}_{\pi^{0 *}} \gamma \gamma^{*}$ can be found in ref. [34]. When applied to the $\mathrm{C} \chi \mathrm{QM}$ we have:

$$
\begin{aligned}
& a_{\mu}^{\mathrm{HLbyL}}\left(\pi^{0}\right)_{\chi \mathrm{QM}} \\
&=e^{2} \frac{8}{3} \int \frac{d^{4} q_{1}}{(2 \pi)^{4}} \int \frac{d^{4} q_{2}}{(2 \pi)^{4}} \frac{1}{q_{1}^{2} q_{2}^{2}\left(q_{1}+q_{2}\right)^{2}\left[q_{1}^{2}+2 p \cdot q_{1}\right]\left[q_{2}^{2}-2 p \cdot q_{2}\right]} \\
& \times {\left[\begin{array}{l}
T_{1}\left(q_{1}, q_{2} ; p\right) \frac{\mathcal{F}_{\pi^{0 *} \gamma^{*} \gamma^{*}}^{(\mathrm{QM})}\left(q_{2}^{2}, q_{1}^{2},\left(q_{1}+q_{2}\right)^{2}\right) \mathcal{F}_{\pi^{*} \gamma^{*} \gamma^{*}}^{(\chi \mathrm{QM})}\left(q_{2}^{2}, q_{2}^{2}, 0\right)}{q_{2}^{2}-m_{\pi}^{2}} \\
+
\end{array}\right.} \\
&\left.+T_{2}\left(q_{1}, q_{2} ; p\right) \frac{\mathcal{F}_{\pi^{0^{*}} \gamma^{*} \gamma^{*}}^{(\chi \mathrm{QM})}\left(\left(q_{1}+q_{2}\right)^{2}, q_{1}^{2}, q_{2}^{2}\right) \mathcal{F}_{\pi^{*} \gamma^{*} \gamma^{*}}^{(\chi \mathrm{QM})}\left(\left(q_{1}+q_{2}\right)^{2},\left(q_{1}+q_{2}\right)^{2}, 0\right)}{\left(q_{1}+q_{2}\right)^{2}-m_{\pi}^{2}}\right],
\end{aligned}
$$

where

$$
\begin{aligned}
T_{1}\left(q_{1}, q_{2} ; p\right)= & 2\left(p \cdot q_{1}\right)\left(p \cdot q_{2}\right)\left(q_{1} \cdot q_{2}\right)-2\left(p \cdot q_{2}\right)^{2} q_{1}^{2}-\left(p \cdot q_{1}\right)\left(q_{1} \cdot q_{2}\right) q_{2}^{2} \\
& +3\left(p \cdot q_{2}\right) q_{1}^{2} q_{2}^{2}-2\left(p \cdot q_{2}\right)\left(q_{1} \cdot q_{2}\right)^{2}+2 m_{\mu}^{2}\left[q_{1}^{2} q_{2}^{2}-\left(q_{1} \cdot q_{2}\right)^{2}\right]
\end{aligned}
$$

originates in the first and second diagrams of figure 12, which give identical contributions, while

$$
\begin{aligned}
T_{2}\left(q_{1}, q_{2} ; p\right)= & 2\left(p \cdot q_{1}\right)\left(p \cdot q_{2}\right)\left(q_{1} \cdot q_{2}\right)-2\left(p \cdot q_{1}\right)^{2} q_{2}^{2}+\left(p \cdot q_{1}\right)\left(q_{1} \cdot q_{2}\right) q_{2}^{2} \\
& +\left(p \cdot q_{1}\right) q_{1}^{2} q_{2}^{2}+m_{\mu}^{2}\left[q_{1}^{2} q_{2}^{2}-\left(q_{1} \cdot q_{2}\right)^{2}\right]
\end{aligned}
$$

originates in the third diagram of figure 12 .

It is well known [35] that, asymptotically for $M_{Q} \gg m_{\pi}$, the $\pi^{0}$-exchange contribution must behave as:

$$
a_{\mu}^{\mathrm{HLbyL}}\left(\pi^{0}\right)_{\chi \mathrm{QM}}=\left(\frac{\alpha}{\pi}\right)^{3} N_{c}^{2} \frac{m_{\mu}^{2}}{16 \pi^{2} f_{\pi}^{2}}\left[\frac{1}{3} \ln ^{2} \frac{M_{Q}}{m_{\pi}}+\mathcal{O}\left(\ln \frac{M_{Q}}{m_{\pi}}\right)+\mathcal{O}(\text { cte. })\right] .
$$

In the $\mathrm{C} \chi \mathrm{QM}$ this contribution can be evaluated exactly. Notice that $\mathcal{F}_{\pi^{0 *} \gamma^{*} \gamma^{*}}^{(\chi \mathrm{QM})}\left(q^{2}, q^{2}, 0\right)$ has a simple analytic expression:

$$
\begin{aligned}
\mathcal{F}_{\pi^{0 *} \gamma^{*} \gamma^{*}}^{(\chi \mathrm{QM})}\left(q^{2}, q^{2}, 0\right) & =-i e^{2} \frac{\mathrm{N}_{\mathrm{c}}}{12 \pi^{2} f_{\pi}} \int_{0}^{1} d x \frac{M_{Q}^{2}}{M_{Q}^{2}-x(1-x) q^{2}} \\
& =i e^{2} \frac{\mathrm{N}_{\mathrm{c}}}{12 \pi^{2} f_{\pi}}\left[\frac{M_{Q}^{2}}{Q^{2}} \frac{2}{\sqrt{1+\frac{4 M_{Q}^{2}}{Q^{2}}}} \log \frac{\sqrt{1+\frac{4 M_{Q}^{2}}{Q^{2}}}-1}{\sqrt{1+\frac{4 M_{Q}^{2}}{Q^{2}}}+1}\right] \\
& =-i e^{2} \frac{\mathrm{N}_{\mathrm{c}}}{12 \pi^{2} f_{\pi}} \frac{1}{2 \pi i} \int_{c_{s}-i \infty}^{c_{s}+i \infty} d s\left(\frac{q^{2}}{\left[-M_{Q}^{2}\right]}\right)^{-s} \frac{\Gamma(s) \Gamma(1-s)^{3}}{\Gamma(2-2 s)},
\end{aligned}
$$


where the expression in the third line gives a very useful representation for analytic evaluations. The full expression of the other vertex function is:

$$
\begin{aligned}
\mathcal{F}_{\pi^{*} \gamma^{*} \gamma^{*}}^{(\chi \mathrm{QM})} & \left(q_{2}^{2}, q_{1}^{2}, q_{3}^{2}\right)=-i e^{2} \frac{N_{c}}{12 \pi^{2} f_{\pi}} \times \\
& \times \int_{0}^{1} d x x \int_{0}^{1} d y \frac{2 M_{Q}^{2}}{M_{Q}^{2}-x(1-x)(1-y) q_{1}^{2}-x^{2} y(1-y) q_{2}^{2}-x y(1-x) q_{3}^{2}},
\end{aligned}
$$

for which the following representation can also be used:

$$
\begin{aligned}
& \mathcal{F}_{\pi^{0^{*} \gamma^{*} \gamma^{*}}}^{(\mathrm{QM})}\left(q_{2}^{2}, q_{1}^{2}, q_{3}^{2}\right)=-i e^{2} \frac{N_{c}}{12 \pi^{2} f_{\pi}} \times \\
& \quad \times\left(\frac{1}{2 \pi i}\right)^{3} 2 \int_{c_{1}-i \infty}^{c_{1}+i \infty} d s_{1}\left(\frac{q_{1}^{2}}{\left[-M_{Q}^{2}\right]}\right)^{-s_{1} c_{2}+i \infty} \int_{c_{2}-i \infty}^{+\infty} d s_{2}\left(\frac{q_{2}^{2}}{\left[-M_{Q}^{2}\right]}\right)^{-s_{2} c_{3}+i \infty} \int_{c_{3}-i \infty}^{-i_{\infty}} d s_{3}\left(\frac{q_{3}^{2}}{\left[-M_{Q}^{2}\right]}\right)^{-s_{3}} \times \\
& \quad \times \frac{\Gamma\left(1-s_{1}-s_{2}\right) \Gamma\left(1-s_{1}-s_{3}\right) \Gamma\left(1-s_{2}-s_{3}\right)}{\Gamma\left(3-2 s_{1}-2 s_{2}-2 s_{3}\right)} \Gamma\left(s_{1}\right) \Gamma\left(s_{2}\right) \Gamma\left(s_{3}\right) \Gamma\left(1-s_{1}-s_{2}-s_{3}\right) .
\end{aligned}
$$

The interest of this Mellin-Barnes representation is that it only modifies the powers of the propagators: $q_{1}^{2}, q_{2}^{2}$ and $\left(q_{1}+q_{2}\right)^{2}$ in the first line of eq. (4.6), and it provides a systematic way ${ }^{8}$ to compute the asymptotic expansion in $\frac{m_{\mu}^{2}}{M_{Q}^{2}}$ and $\frac{m_{\mu}^{2}}{m_{\pi}^{2}}$ powers, and powers of logarithms. We postpone, however, this analytic calculation to a forthcoming publication and, instead, proceed here to a numerical evaluation.

In order to evaluate $a_{\mu}^{\mathrm{HLbyL}}\left(\pi^{0}\right)_{\chi \mathrm{QM}}$ in eq. (4.6) numerically, it is useful to apply to the integrand in that equation the technique of Gegenbauer polynomial expansion, as was done in ref. [34]. Then one can reduce the $q_{1}$ and $q_{2}$ integrations to two euclidean integrals over $Q_{1}^{2} \equiv-q_{1}^{2}$ and $Q_{2}^{2} \equiv-q_{2}^{2}$ ( both from 0 to $\infty$ ), and an integral over $\cos \theta$ with $\theta$ the angle between the two euclidean four-vectors $Q_{1}$ and $Q_{2}$. The integrand in question, which is explicitly given in ref. [3], is then very convenient for numerical integration.

We find

$$
a_{\mu}^{\mathrm{HLbyL}}\left(\pi^{0}\right)_{\chi \mathrm{QM}}=68.0 \times 10^{-11} \text { for } \quad M_{Q}=240 \mathrm{MeV},
$$

with a range

$$
a_{\mu}^{\mathrm{HLbyL}}\left(\pi^{0}\right)_{\chi \mathrm{QM}}=64.6 \times 10^{-11} \text { for } \quad M_{Q}=230 \mathrm{MeV},
$$

and

$$
a_{\mu}^{\mathrm{HLbyL}}\left(\pi^{0}\right)_{\chi \mathrm{QM}}=71.3 \times 10^{-11} \quad \text { for } \quad M_{Q}=250 \mathrm{MeV} .
$$

The result

$$
a_{\mu}^{\mathrm{HLbyL}}\left(\pi^{0}\right)_{\chi \mathrm{QM}}=(68 \pm 3) \times 10^{-11},
$$

which does not include the systematic error of the model, agrees well with the phenomenological determinations of this contribution which, according to the most recent update [38] and depending on the underlying phenomenological model for the form factors $\mathcal{F}_{\pi^{0 *} \gamma^{*} \gamma^{*}}^{(\chi \mathrm{QM})}\left(q_{2}^{2}, q_{1}^{2}, q_{3}^{2}\right)$ vary between

$$
a_{\mu}^{\mathrm{HLbyL}}\left(\pi^{0}\right)_{\text {phen. }}=(57.4 \pm 4.6) \times 10^{-11} \quad \text { and } \quad a_{\mu}^{\mathrm{HLbyL}}\left(\pi^{0}\right)_{\text {phen. }}=(80.1 \pm 4.7) \times 10^{-11} .
$$

\footnotetext{
${ }^{8}$ See e.g. ref. [36] for an example of this method.
} 
Again, for the same reasons mentioned at the end of section 3.4.1, we do not discuss here the contributions from the $\eta$ and $\eta^{\prime}$ exchanges.

It is a fact that asymptotically, for $M_{Q} \rightarrow \infty$, the $\pi^{0}$ contribution largely dominates the Constituent Quark Loop contribution:

$$
\left.\frac{a_{\mu}^{\mathrm{HLbyL}}\left(\pi^{0}\right)}{a_{\mu}^{\mathrm{HLbyL}}(\mathrm{CQL})}\right|_{M_{Q} \rightarrow \infty} \sim \frac{N_{c} M_{Q}^{2}}{16 \pi^{2} f_{\pi}^{2}} \frac{1}{\zeta(3)-\frac{19}{24}} \log ^{2} \frac{M_{Q}}{m_{\pi}} ;
$$

however, this asymptotic behaviour is far from being reached at values of $M_{Q}$ between $230 \mathrm{MeV}$ and $250 \mathrm{MeV}$, for which the Constituent Quark Loop contribution still dominates over the Goldstone contribution.

For the total hadronic light-by-light contribution in the $\mathrm{C} \chi \mathrm{QM}$, which includes the quark loop contribution as well as the $\pi^{0}$-exchange contributions, we then find

$$
148 \times 10^{-11} \leq a_{\mu}^{(\mathrm{HLbyL})}(\mathrm{C} \chi \mathrm{QM}) \leq 153 \times 10^{-11}, \quad \text { for } \quad 250 \mathrm{MeV} \geq M_{Q} \geq 230 \mathrm{MeV} .
$$

This result, which does not include the systematic error of the model, has to be compared with the phenomenological estimate

$$
a^{(\mathrm{HLbyL})}=(122 \pm 18) \times 10^{-11},
$$

for the total of the hadronic contributions not suppressed in the $1 / \mathrm{N}_{\mathrm{c}}$-expansion (see e.g. ref. [5] for details). Within the expected systematic uncertainties they compare rather well.

The interesting feature which emerges from this calculation is the observed balance between the Goldstone contribution and the Quark Loop contribution. Indeed, as the constituent quark mass $M_{Q}$ gets larger and larger, the Goldstone contribution dominates; while for $M_{Q}$ smaller and smaller it is the Quark Loop contribution which dominates. This is illustrated by the plot of the total $a_{\mu}^{(\mathrm{HLbyL})}(\mathrm{C} \chi \mathrm{QM})$ versus $M_{Q}$ shown in figure 14 . What this plot shows is in flagrant contradiction with the results reported in ref. [39] based in a calculation using a Dyson-Schwinger inspired model. In this model, the authors find a contribution from the $\pi^{0}$-exchange which, within errors, is compatible with the other phenomenological determinations and, in particular, with our $\mathrm{C} \chi \mathrm{QM}$ result in eq. (4.16); yet their result for the equivalent contribution to the quark loop turns out to be almost twice as large with a total contribution

$$
a^{(\text {HLbyL })}(\text { ref. [39] })=(217 \pm 91) \times 10^{-11} .
$$

The central value of this result would require a ridicously small value of $M_{Q}$ in order to be reproduced by the $\mathrm{C} \chi \mathrm{QM}$ and, furthermore, for such a small value of $M_{Q}$ the $\pi^{0}$-exchange contribution would be far too small as compared to all the phenomenological estimates, including the one in ref. [39]. We conclude that a range of values such as

$$
170 \leq a_{\mu}^{(\mathrm{HLbyL})} \times 10^{11} \leq 308,
$$

allowed by the result quoted in eq. (4.21), cannot be digested within the $\mathrm{C} \chi \mathrm{QM}$ and in our opinion this casts serious doubts about the compatibility of the model used in ref. [39] with basic QCD features. 


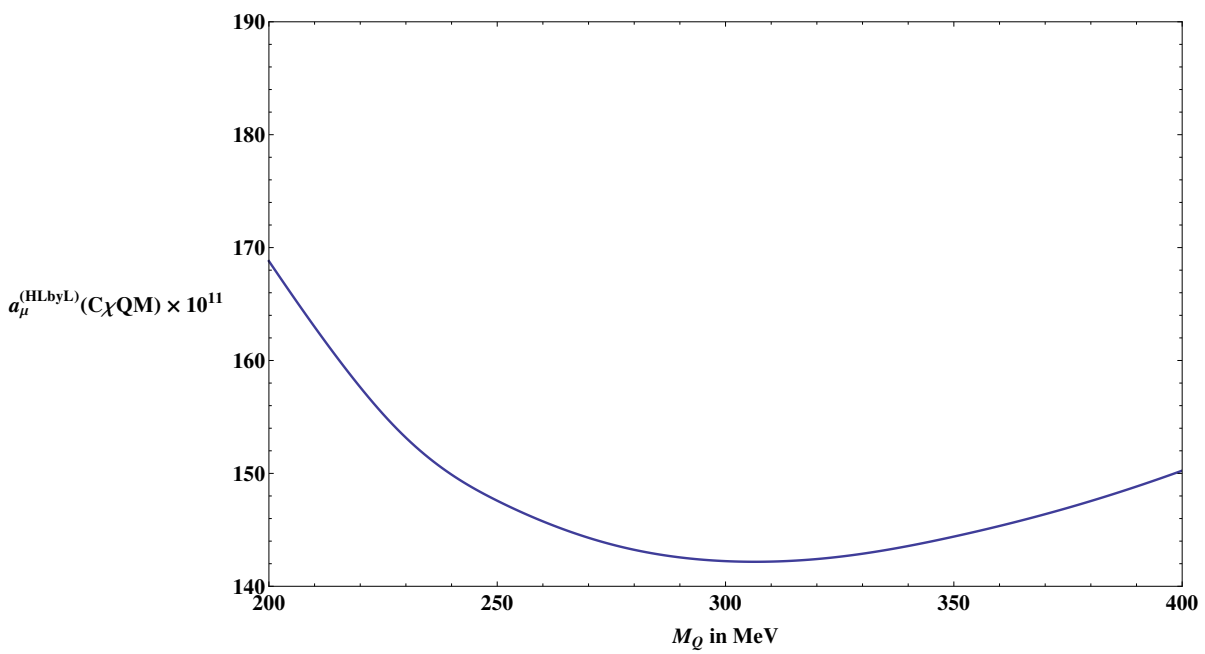

Figure 14. $a_{\mu}^{(\mathrm{HLbyL})}(\mathrm{C} \chi \mathrm{QM})$ versus $M_{Q}$ in in $10^{-11}$ units.
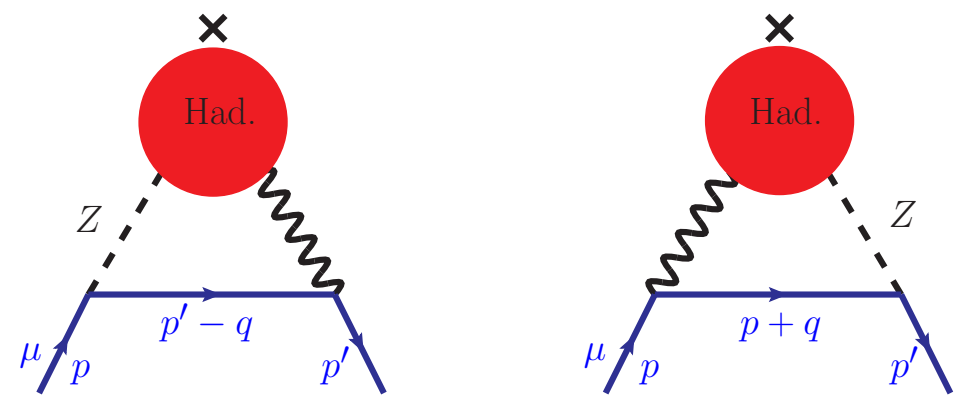

Figure 15. Feynman diagrams with the hadronic $\gamma \gamma Z$ vertex which contributes to the muon anomaly.

\section{$5 \quad$ Hadronic electroweak contributions}

These are the contributions to the muon anomaly which appear at the two-loop level in the electroweak sector. They are the ones generated by the hadronic $\gamma \gamma Z$ vertex, with one $\gamma$ and the $Z$-boson attached to a muon line, as illustrated by the Feynman diagrams in figure 15 .

These contributions are particularly interesting because, a priori, they could be enhanced by a large $\log \left(M_{Z}^{2} / m_{\mu}^{2}\right)$ factor. However, due to the anomaly-free coupling assignments in the Standard Model, there is an important cancellation of UV-scales between the lepton and the quark contributions within a given family [40,41]. What is left out of this cancellation in the sector of the $u, d$ and $s$ quarks, where the strong interactions play a subtle role at long distances, is governed by the dynamics of spontaneous chiral symmetry breaking [40, 42-45]. The C $\chi \mathrm{QM}$, where the hadronic blob in figure 15 is replaced by a constituent quark loop as illustrated in figure 16, offers a simple way to estimate these contributions which we next discuss. 
In full generality, the hadronic $\gamma \gamma Z$ contribution to the muon anomly, which we denote by $a_{\mu}^{\mathrm{HEW}}$ is given by the following representation [42]:

$$
\begin{aligned}
a_{\mu}^{(\mathrm{HEW})}=\left(i e^{2}\right) & \frac{g^{2}}{16 \cos ^{2} \theta_{W}} \frac{1}{M_{Z}^{2}} \lim _{k^{2} \rightarrow 0} \int \frac{d^{4} q}{(2 \pi)^{4}} \frac{1}{q^{2}}\left(\frac{M_{Z}^{2}}{q^{2}-M_{Z}^{2}}\right) \times \\
& \times \frac{1}{4 k^{2}} \operatorname{tr}\left\{\left(\not p+m_{\mu}\right)\left[\gamma^{\rho} \not k-\left(k^{\rho}+\frac{p^{\rho}}{m_{\mu}} \not k\right)\right] \times\right. \\
& \left.\times\left[\gamma^{\mu} \frac{\left(\not p-\not q+m_{\mu}\right)}{q^{2}-2 q \cdot p} \gamma^{\nu} \gamma_{5}+\gamma^{\nu} \gamma_{5} \frac{\left(\not p+\not q+m_{\mu}\right)}{q^{2}+2 q \cdot p} \gamma^{\mu}\right]\right\} W_{\mu \nu \rho}(q, k),
\end{aligned}
$$

where $W_{\mu \nu \rho}(q, k)$ denotes the hadronic Green's function:

$$
W_{\mu \nu \rho}(q, k)=\int d^{4} x e^{i q \cdot x} \int d^{4} y e^{i(k-q) \cdot y}\left\langle 0\left|T\left\{V_{\mu}^{\mathrm{em}}(x) A_{\nu}^{\mathrm{nc}}(y) V_{\rho}^{\mathrm{em}}(0)\right\}\right| 0\right\rangle,
$$

with $k$ the incoming photon four-momentum associated with the classical external magnetic field, and where

$$
V_{\mu}^{\mathrm{em}}(x)=\bar{q}(x) \gamma_{\mu} Q q(x), \quad \text { and } \quad A_{\nu}^{\mathrm{nc}}(y)=\bar{q}(y) \gamma_{\nu} \gamma_{5} Q_{L}^{(3)}, q(y)
$$

with

$$
Q=Q_{L}=Q_{R}=\operatorname{diag}(2 / 3,-1 / 3,-1 / 3), \quad \text { and } \quad Q_{L}^{(3)}=\operatorname{diag}(1,-1,-1) .
$$

The relevant question here is the contribution to $a_{\mu}^{(\mathrm{HEW})}$ from the non-anomalous part of $W_{\mu \nu \rho}(q, k)$, denoted by $\tilde{W}_{\mu \nu \rho}(q, k)$, i.e.

$$
W_{\mu \nu \rho}(q, k)=-i \frac{N_{c}}{12 \pi^{2}} \frac{4}{3} \frac{(q-k)_{\nu}}{(q-k)^{2}} \epsilon_{\mu \rho \alpha \beta} q^{\alpha} k^{\beta}+\tilde{W}_{\mu \nu \rho}(q, k),
$$

where the first term in the r.h.s. is the one generated by the VVA anomaly. The second term $\tilde{W}_{\mu \nu \rho}(q, k)$, in the chiral limit where the light quark masses are neglected, is then fully transverse in the axial neutral current ( $\nu$ index) and the Ward identities constrain it to have the form $\left(Q^{2}=-q^{2}\right)[42]$ :

$$
\tilde{W}_{\mu \nu \rho}(q, k)=i k^{\sigma}\left[q_{\rho} \epsilon_{\mu \nu \alpha \sigma} q^{\alpha}-q_{\sigma} \epsilon_{\mu \nu \alpha \rho} q^{\alpha}\right] W\left(Q^{2}\right),
$$

with only one invariant function $W\left(Q^{2}\right)$ which depends on the details of the dynamics.

In the $\mathrm{C} \chi \mathrm{QM}$, with $g_{A}=1$, the function $W\left(Q^{2}\right)$ is given by the expression:

$$
\begin{aligned}
W_{\chi \mathrm{QM}}\left(Q^{2}\right) & =\frac{N_{c}}{12 \pi^{2}} \frac{8}{3} \frac{1}{M_{Q}^{2}} \times \int_{0}^{1} d x \int_{0}^{1-x} d y \frac{x y-y(1-y)}{1+\frac{Q^{2}}{M_{Q}^{2}} y(1-y)} \\
& =\frac{-N_{c}}{12 \pi^{2}} \frac{2}{3} \frac{1}{Q^{2}}\left\{1+\frac{M_{Q}^{2}}{Q^{2}} \frac{2}{\sqrt{1+\frac{4 M_{Q}^{2}}{Q^{2}}}} \log \frac{\sqrt{1+\frac{4 M_{Q}^{2}}{Q^{2}}}-1}{\sqrt{1+\frac{4 M_{Q}^{2}}{Q^{2}}}+1}\right\} .
\end{aligned}
$$

Not surprisingly, the second term in the brackets coincides with the analytic expression of the $\mathrm{C} \chi \mathrm{QM}$ vertex function given by the term in brackets in the second line of 


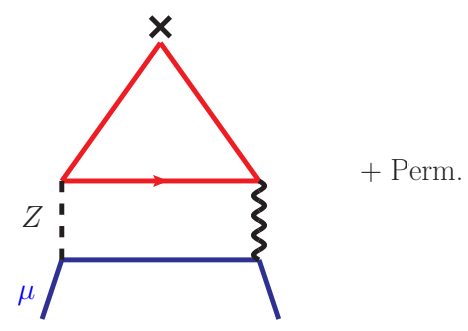

Figure 16. Feynman diagrams in the $\mathrm{C} \chi \mathrm{QM}$ of the $\gamma \gamma Z$ vertex type.

eq. (4.10). This is because, up to an overall factor, $W_{\chi \mathrm{QM}}\left(Q^{2}\right)$ is precisely the same function as $\mathcal{F}_{\pi^{0} \gamma^{*} \gamma^{*}}^{(\chi \mathrm{QM})}\left(q^{2}, q^{2}, 0\right)$ with the anomaly $\left(M_{Q} \rightarrow \infty\right)$ subtracted. It is this fact that guarantees that $W_{\chi \mathrm{QM}}\left(Q^{2}\right)$, for $g_{A}=1$, has the correct pQCD leading short-distance behaviour [43, 45]:

$$
W_{\chi \mathrm{QM}}\left(Q^{2}\right) \underset{Q^{2} \rightarrow \infty}{\longrightarrow} \frac{-N_{c}}{12 \pi^{2}} \frac{2}{3} \frac{1}{Q^{2}} .
$$

The $\mathrm{C} \chi \mathrm{QM}$, however, has its limitations and does not predict correctly the subleading short-distance behaviour:

$$
\lim _{Q^{2} \rightarrow \infty} W_{\chi \mathrm{QM}}\left(Q^{2}\right)=\frac{-N_{c}}{12 \pi^{2}} \frac{2}{3}\left[\frac{1}{Q^{2}}-2 \frac{M_{Q}^{2}}{Q^{4}} \log \frac{Q^{2}}{M_{Q}^{2}}+\mathcal{O}\left(\frac{M_{Q}^{4}}{Q^{6}} \log \frac{Q^{2}}{M_{Q}^{2}}\right)\right],
$$

which falls as $\mathcal{O}\left(\frac{1}{Q^{4}}\right)$, while the OPE in QCD predicts that this subleading term must fall as $\mathcal{O}\left(\frac{1}{Q^{6}}\right)[42]$.

Another interesting limit is the long-distance behaviour of the function $W\left(Q^{2}\right)$ which in QCD is related to a coupling constant of $\mathcal{O}\left(p^{6}\right)$ in the odd-parity sector of the effective chiral Lagrangian [45]. Here, the prediction of the $\mathrm{C} \chi \mathrm{QM}$ is

$$
W_{\chi \mathrm{QM}}\left(Q^{2}\right) \underset{Q^{2} \rightarrow 0}{\longrightarrow} \frac{-N_{c}}{12 \pi^{2}} \frac{1}{9 M_{Q}^{2}} .
$$

Unfortunately, there is no model independent prediction for $W(0)$ to compare with.

The contribution to $a_{\mu}^{(\mathrm{HEW})}$ from the anomalous term in eq. (5.5), evaluated in the Feynman gauge $[40,42]$ is:

$$
\begin{aligned}
\left.a_{\mu}^{(\mathrm{HEW})}\right|_{\text {anom }} & =\frac{G_{\mathrm{F}}}{\sqrt{2}} \frac{m_{\mu}^{2}}{8 \pi^{2}} \frac{\alpha}{\pi} \frac{N_{c}}{3}\left\{\frac{4}{3} \log \frac{M_{Z}^{2}}{m_{\mu}^{2}}+\frac{2}{3}+\mathcal{O}\left(\frac{m_{\mu}^{2}}{M_{Z}^{2}} \log \frac{M_{Z}^{2}}{m_{\mu}^{2}}\right)\right\} \\
& =\frac{m_{\mu}^{2}}{8 \pi^{2}} \frac{\alpha}{\pi} \times 18.69
\end{aligned}
$$

and the one from the transverse component $\tilde{W}_{\mu \nu \rho}(q, k)$ in eq. (5.5), evaluated in the $\mathrm{C} \chi \mathrm{QM}$ in the Feynman gauge and with $g_{A}=1$ :

$$
\left.a_{\mu}^{(\mathrm{HEW})}\right|_{\text {transv }} ^{\chi \mathrm{QM}}=\frac{G_{\mathrm{F}}}{\sqrt{2}} \frac{m_{\mu}^{2}}{8 \pi^{2}} \frac{\alpha}{\pi} \times \frac{N_{c}}{3}\left[\frac{2}{3} \log \frac{M_{Z}^{2}}{M_{Q}^{2}}-\frac{4}{3}+\mathcal{O}\left(\frac{M_{Q}^{2}}{M_{Z}^{2}} \log \frac{M_{Z}^{2}}{M_{Q}^{2}}\right) .\right] .
$$


The sum of these two contributions takes care of the hadronic sector induced by the dynamics of the light quarks $u, d$ and $s$; but, as already mentioned, it is only when added to the lepton contributions from the electron and the muon and the one from the heavy charm quark that the whole sum is gauge independent and it then makes sense in the Standard Model. We reproduce below the details of this overall result:

$$
\begin{aligned}
& \left.a_{\mu}^{(\mathrm{HEW})}\right|_{e, u, d ; \mu, s, c}=\frac{G_{\mathrm{F}}}{\sqrt{2}} \frac{m_{\mu}^{2}}{8 \pi^{2}} \frac{\alpha}{\pi} \times \\
& \times\{\underbrace{-3 \log \frac{M_{Z}^{2}}{m_{\mu}^{2}}-\frac{5}{2}}_{\text {electron }}+\underbrace{2 \log \frac{M_{Z}^{2}}{m_{\mu}^{2}}+1}_{\mathrm{u}, \mathrm{d} \text { anom }}+\underbrace{\log \frac{M_{Z}^{2}}{M_{Q}^{2}}-2}_{\mathrm{u}, \mathrm{d} \text { transv } \mathrm{C} \chi \mathrm{QM}} \\
& \underbrace{-3 \log \frac{M_{Z}^{2}}{m_{\mu}^{2}}-\frac{11}{6}+\frac{8}{9} \pi^{2}}_{\text {muon }} \underbrace{-\frac{2}{3} \log \frac{M_{Z}^{2}}{m_{\mu}^{2}}-\frac{1}{3}}_{\mathrm{s} \text { anom }} \underbrace{-\frac{1}{3} \log \frac{M_{Z}^{2}}{M_{Q}^{2}}+\frac{2}{3}}_{\mathrm{s} \text { transv } \mathrm{C} \chi \mathrm{QM}} \underbrace{+4 \log \frac{M_{Z}^{2}}{m_{c}^{2}}}_{\text {charm }}\} .
\end{aligned}
$$

Notice how the $\log M_{Z}^{2}$ dependence cancels in each generation [44], and we finally obtain

$$
\begin{aligned}
a_{\mu}^{\left.(\mathrm{HEW})\right|_{e, u, d ; \mu, s, c}(\mathrm{C} \chi \mathrm{QM})} & =\frac{G_{\mathrm{F}}}{\sqrt{2}} \frac{m_{\mu}^{2}}{8 \pi^{2}} \frac{\alpha}{\pi}\left(-\frac{2}{3} \log \frac{M_{Q}^{2}}{m_{\mu}^{2}}-4 \log \frac{m_{c}^{2}}{m_{\mu}^{2}}-5+\frac{8}{9} \pi^{2}\right) \\
& =-\frac{G_{\mathrm{F}}}{\sqrt{2}} \frac{m_{\mu}^{2}}{8 \pi^{2}} \frac{\alpha}{\pi} \times 18.6=-5.0 \times 10^{-11},
\end{aligned}
$$

for $m_{c}=1.5 \mathrm{GeV}$ and $M_{Q}=240 \mathrm{MeV}$, a result which within the systematic errors of the model, is compatible with the phenomenological determination [44]:

$$
\left.a_{\mu}^{(\mathrm{HEW})}\right|_{e, u, d ; \mu, s, c}=(-6.7 \pm 0.5) \times 10^{-11} .
$$

\section{Summary and conclusions}

From the previous considerations we conclude that the $\mathrm{C} \chi \mathrm{QM}$ provides a useful and simple reference model to evaluate the hadronic contributions to the anomalous magnetic moment of the muon. The effective Lagrangian of this model is renormalizable in the Large- $\mathrm{N}_{\mathrm{c}}$ limit [12] and, as shown in [11], the number of the required counterterms in this limit is minimized for a choice of the axial coupling: $g_{A}=1$. The only free parameter of the model is then the mass of the constituent quark mass $M_{Q}$ which in section 2, from a comparison with the phenomenological determination of the lowest order hadronic vacuum polarization contribution to the muon anomaly, has been fixed to

$$
M_{Q}=(240 \pm 10) \mathrm{MeV},
$$

This range of values for $M_{Q}$ reproduces the phenomenological determination within an error of less than $10 \%$. All the other hadronic contributions have then been evaluated for this range of values of $M_{Q}$ with the results which are summarized in table 2. 


\begin{tabular}{|c|c|}
\hline Class & Result in $10^{-10}$ units \\
\hline HVP & $652+47$ \\
& -42 \\
HVP to $\mathcal{O}\left(\frac{\alpha}{\pi}\right)^{3}$ & $-6.4 \pm 1.2$ \\
HLbyL & $15.0 \pm 0.3$ \\
HEW & -0.5 \\
\hline
\end{tabular}

Table 2. Summary of results for the hadronic contributions to the muon anomly in the $\mathrm{C} \chi \mathrm{QM}$.

We want to emphasize that the errors quoted in table 2 are only those generated by the error of $M_{Q}$ in eq. (2.15) and they do not reflect the systematic error of the model. These results, within a systematic error of $20 \%$ to $30 \%$, are in good agreement with the phenomenological determinations. One exception, discussed in detail in section 3.4.1, is the contribution from the $\pi^{0} \gamma$ intermediate state to hadronic vacuum polarization where the $\mathrm{C} \chi \mathrm{QM}$, because of its $\mathrm{SU}(3)$ invariance, fails to reproduce the phenomenological determination which is particularly enhanced because of the large observed branching ratio in eq. (3.54).

Ironically, the error \pm 0.3 for the Hadronic Light-by-Light contribution appears to be the smallest relative error. This is due to the fact that, as shown in figure 16, the sum of the quark loop contribution and the Goldstone exchange contribution for values of $M_{Q}$ in the range of eq. (2.15) is already very near to the minimum in the $M_{Q}$-dependence of the sum of these two contributions, which occurs at $M_{Q} \simeq 300 \mathrm{MeV}$. In other words, in the $\mathrm{C} \chi \mathrm{QM}$ the contribution to the muon anomaly which is less sensitive to the value of the constituent quark mass is precisely the one from the hadronic light-by-light scattering. This fact, however, should not mask the intrinsic systematic error which has not been included. Within an expected systematic error of $\sim 20 \%$, our results agree with the phenomenological determinations reviewed in ref. [5]. An exception, however, is the determination quoted in eq. (4.21). As discussed in the text, the large range of values allowed by this result, cannot be digested within the $\mathrm{C} \chi \mathrm{QM}$ and in our opinion casts serious doubts about the compatibility of the model used in ref. [39] with basic QCD features.

Another interesting feature, which has appeared when evaluating the hadronic electroweak contributions, is the impact of the choice $g_{A}=1$, which was initially made on theoretical grounds. It turns out that it is only for this choice that the $\mathrm{C} \chi \mathrm{QM}$ has the correct matching at short-distances with the one predicted by the OPE in QCD [43, 45] when evaluating the hadronic electroweak contribution.

Concerning the next-to-leading contributions from Hadronic Vacuum Polarization we have made two observations which are in fact model independent. On the one hand we have explained why this contribution is smaller than the naive expected order of magnitude and on the other hand we have derived a sum rule in eq. (3.27) which offers an interesting constraint when evaluating radiative corrections. 


\section{Acknowledgments}

We wish to thank Marc Knecht for many helpful discussions on the topics discussed in this paper. We thank Marc Knecht, Santi Peris and Laurent Lellouch for a careful reading of the manuscript

The work of DG has been supported by MICINN (grant FPA2009-09638) and DGIIDDGA (grant 2009-E24/2) and by the Spanish Consolider-Ingenio 2010 Program CPAN (CSD2007-00042).

Open Access. This article is distributed under the terms of the Creative Commons Attribution License which permits any use, distribution and reproduction in any medium, provided the original author(s) and source are credited.

\section{References}

[1] Muon G-2 collaboration, G. Bennett et al., Final report of the muon E821 anomalous magnetic moment measurement at BNL, Phys. Rev. D 73 (2006) 072003 [hep-ex/0602035] [INSPIRE].

[2] J.P. Miller, E. de Rafael and B.L. Roberts, Muon $(g-2)$ : experiment and theory, Rept. Prog. Phys. 70 (2007) 795.

[3] F. Jegerlehner and A. Nyffeler, The muon g-2, Phys. Rept. 477 (2009) 1 [arXiv: 0902.3360] [INSPIRE].

[4] M. Davier, A. Hoecker, B. Malaescu and Z. Zhang, Reevaluation of the hadronic contributions to the muon $g-2$ and to $\alpha_{M Z}$, Eur. Phys. J. C 71 (2011) 1515 [Erratum ibid. C 72 (2012) 1874] [arXiv:1010.4180] [INSPIRE].

[5] J. Prades, E. de Rafael and A. Vainshtein, The hadronic light-by-light contribution to $a_{\mu, e}$, in Lepton dipole moments, Advanced series on directions in high energy physics volume 20, B.L. Roberts and W.J. Marciano eds., World Scientific, Singapore (2009)

[6] R. Carey et al., FERMILAB-PROPOSAL-0989.

[7] T. Mibe, Measurement of muon $g-2$ and EDM with an ultra-cold muon beam at J-PARC, Nucl. Phys. (Proc. Suppl.) B 218 (2011) 242.

[8] A.A. Andrianov and L. Bonora, Finite-mode regularization of the fermion functional integral, Nucl. Phys. B 233 (1984) 232 [inSPIRE].

[9] A. De Rújula, H. Georgi and S. Glashow, Hadron masses in a gauge theory, Phys. Rev. D 12 (1975) 147 [inSPIRE].

[10] S. Weinberg, Phenomenological lagrangians, Physica 96A (1984) 327.

[11] E. de Rafael, The constituent chiral quark model revisited, Phys. Lett. B 703 (2011) 60 [arXiv: 1107.0226] [INSPIRE].

[12] S. Weinberg, Pions in large- $N$ quantum chromodynamics, Phys. Rev. Lett. 105 (2010) 261601 [arXiv:1009.1537] [INSPIRE].

[13] C. Bouchiat and L. Michel, La résonance dans la diffusion méson $\pi^{-}$méson $\pi$ et le moment magnétique anormal du méson $\mu, J$. Phys. Radium 22 (1961) 121. 
[14] S.J. Brodsky and E. De Rafael, Suggested boson-lepton pair couplings and the anomalous magnetic moment of the muon, Phys. Rev. 168 (1968) 1620 [INSPIRE].

[15] J. Calmet, S. Narison, M. Perrottet and E. de Rafael, The anomalous magnetic moment of the muon: a review of the theoretical contributions, Rev. Mod. Phys. 49 (1977) 21 [INSPIRE].

[16] A. Pivovarov, Muon anomalous magnetic moment: a consistency check for the next-to-leading order hadronic contributions, Phys. Atom. Nucl. 66 (2003) 902 [hep-ph/0110248] [INSPIRE].

[17] J. Erler and G.T. Sanchez, An upper bound on the hadronic light-by-light contribution to the muon g-2, Phys. Rev. Lett. 97 (2006) 161801 [hep-ph/0605052] [INSPIRE].

[18] G. Källen and A. Sabry, Fourth order vacuum polarization, Kgl. Danske Videnskab. Selskab, Mat. Fys. Medd. 29N17 (1955) 17.

[19] B. Lautrup and E. De Rafael, Calculation of the sixth-order contribution from the fourth-order vacuum polarization to the difference of the anomalous magnetic moments of muon and electron, Phys. Rev. 174 (1968) 1835 [INSPIRE].

[20] D. Espriu, E. de Rafael and J. Taron, The QCD effective action at long distances, Nucl. Phys. B 345 (1990) 22 [Erratum ibid. B 355 (1991) 278-279] [InSPIRE].

[21] ETMC collaboration, X. Feng, K. Jansen, M. Petschlies and D.B. Renner, Two-flavor QCD correction to lepton magnetic moments at leading-order in the electromagnetic coupling, Phys. Rev. Lett. 107 (2011) 081802 [arXiv:1103.4818] [INSPIRE].

[22] R. Barbieri and E. Remiddi, Electron and muon 1/2 $(g-2)$ from vacuum polarization insertions, Nucl. Phys. B 90 (1975) 233.

[23] K. Hagiwara, A. Martin, D. Nomura and T. Teubner, Predictions for $g-2$ of the muon and $\alpha_{Q E D}\left(M^{2}(Z)\right)$, Phys. Rev. D 69 (2004) 093003 [hep-ph/0312250] [INSPIRE].

[24] B.E. Lautrup, A. Peterman and E. de Rafael, Recent developments in the comparison between theory and experiments in quantum electrodynamics, Phys. Rept. 3C (1972) 193.

[25] E. de Rafael, Hadronic contributions to the muon $g-2$ and low-energy $Q C D$, Phys. Lett. B 322 (1994) 239 [hep-ph/9311316] [InSPIRE].

[26] M. Perrottet and E. de Rafael, unpublished.

[27] I. Blokland, A. Czarnecki and K. Melnikov, Pion pole contribution to hadronic light by light scattering and muon anomalous magnetic moment, Phys. Rev. Lett. 88 (2002) 071803 [hep-ph/0112117] [INSPIRE].

[28] M. Achasov et al., Experimental study of the processes $e^{+} e^{-} \rightarrow \phi \rightarrow \eta \gamma, \pi^{0} \gamma$ at VEPP-2M, Eur. Phys. J. C 12 (2000) 25 [InSPIRE].

[29] K. Hagiwara, R. Liao, A.D. Martin, D. Nomura and T. Teubner, $(g-2)_{\mu}$ and $\alpha\left(M_{Z}^{2}\right)$ re-evaluated using new precise data, J. Phys. G 38 (2011) 085003 [arXiv:1105.3149] [INSPIRE].

[30] F. Jegerlehner, Precision measurements of sigma(hadronic) for $\alpha_{\mathrm{eff}}(E)$ at ILC energies and $(g-2)_{\mu}$, Nucl. Phys. Proc. Suppl. 162 (2006) 22 [hep-ph/0608329] [INSPIRE].

[31] J. Aldins, T. Kinoshita, S.J. Brodsky and A. Dufner, Photon-photon scattering contribution to the sixth order magnetic moment of the muon, Phys. Rev. Lett. 23 (1969) 441 [INSPIRE].

[32] S. Laporta and E. Remiddi, The analytical value of the electron light-light graphs contribution to the muon $(g-2)$ in QED, Phys. Lett. B 301 (1993) 440. 
[33] R. Boughezal and K. Melnikov, Hadronic light-by-light scattering contribution to the muon magnetic anomaly: constituent quark loops and QCD effects, Phys. Lett. B 704 (2011) 193 [arXiv: 1104.4510] [INSPIRE].

[34] M. Knecht and A. Nyffeler, Hadronic light by light corrections to the muon g-2: the pion pole contribution, Phys. Rev. D 65 (2002) 073034 [hep-ph/0111058] [INSPIRE].

[35] M. Knecht, A. Nyffeler, M. Perrottet and E. de Rafael, Hadronic light by light scattering contribution to the muon $g-2$ : an effective field theory approach, Phys. Rev. Lett. 88 (2002) 071802 [hep-ph/0111059] [INSPIRE].

[36] J.-P. Aguilar, D. Greynat and E. De Rafael, Muon anomaly from lepton vacuum polarization and the Mellin-Barnes representation, Phys. Rev. D 77 (2008) 093010 [arXiv:0802.2618] [INSPIRE].

[37] K. Melnikov and A. Vainshtein, Hadronic light-by-light scattering contribution to the muon anomalous magnetic moment revisited, Phys. Rev. D 70 (2004) 113006 [hep-ph/0312226] [INSPIRE].

[38] D. Babusci, H. Czyz, F. Gonnella, S. Ivashyn, M. Mascolo, et al., On the possibility to measure the $\pi^{0}$ to $\gamma \gamma$ decay width and the $\gamma^{*} \gamma$ to $\pi^{0}$ transition form factor with the KLOE-2 experiment, Eur. Phys. J. C 72 (2012) 1917 [arXiv:1109.2461] [INSPIRE].

[39] T. Goecke, C.S. Fischer and R. Williams, Hadronic contribution to the muon $g-2:$ a Dyson-Schwinger perspective, Prog. Part. Nucl. Phys. 67 (2012) 563 [arXiv:1111.0990] [INSPIRE].

[40] S. Peris, M. Perrottet and E. de Rafael, Two loop electroweak corrections to the muon $g-2$ : a new class of hadronic contributions, Phys. Lett. B 355 (1995) 523 [hep-ph/9505405] [INSPIRE].

[41] A. Czarnecki, B. Krause and W. Marciano, Electroweak fermion loop contributions to the muon anomalous magnetic moment, Phys. Rev. D 52 (1995) 2619 [hep-ph/9506256] [INSPIRE].

[42] M. Knecht, S. Peris, M. Perrottet and E. De Rafael, Electroweak hadronic contributions to the muon $(g-2)$, JHEP 11 (2002) 003 [hep-ph/0205102] [INSPIRE].

[43] A. Vainshtein, Perturbative and nonperturbative renormalization of anomalous quark triangles, Phys. Lett. B 569 (2003) 187 [hep-ph/0212231] [INSPIRE].

[44] A. Czarnecki, W.J. Marciano and A. Vainshtein, Refinements in electroweak contributions to the muon anomalous magnetic moment, Phys. Rev. D 67 (2003) 073006 [Erratum ibid. D 73 (2006) 119901] [hep-ph/0212229] [INSPIRE].

[45] M. Knecht, S. Peris, M. Perrottet and E. de Rafael, New nonrenormalization theorems for anomalous three point functions, JHEP 03 (2004) 035 [hep-ph/0311100] [INSPIRE]. 